\title{
THE ANNUAL MEETING OF THE SOCIETY
}

The fifty-seventh Annual Meeting of the American Mathematical Society was held at the University of Florida, Gainesville, Florida, Wednesday through Friday, December 27-29, 1950, in conjunction with the Annual Meeting of the Mathematical Association of Ameri$\mathrm{ca}$, and the Christmas Conference of the National Council of Teachers of Mathematics.

The attendance at the meeting was about 350 , including the following 225 members of the Society:

C. R. Adams, R. B. Adams, O. R. Ainsworth, C. B. Allendoerfer, B. M. Armstrong, Nachman Aronszajn, W. L. Ayres, E. A. Bailey, R. H. Bardell, H. P. Beard, E. G. Begle, R. G. Blake, Laura Blakeley, A. L. Blakers, L. M. Blumenthal, M. I. Blyth, H. W. Bode, F. F. Bonsall, T. A. Botts, Samuel Bourne, G. F. Bradfield, O. E. Brown, C. C. Camp, R. Crowley Campbell, A. B. Carson, E. D. Cashwell, K. T. Chen, Sarvadaman Chowla, K. L. Chung, E. T. Church, C. E. Clark, R. F. Clippinger, Nathaniel Coburn, Eckford Cohen, J. B. Coleman, W. J. Conner, G. M. Conwell, L. P. Copeland, F. E. Cothran, J. C. Currie, P. H. Daus, J. J. Dennis, A. H. Diamond, V. E. Dietrich, L. L. Dines, W. L. Duren, Patrick Du Val, J. C. Eaves, Myrtle Edwards, D. O. Ellis, D. H. Erkiletian, J. L. Evans, W. B. Evans, R. M. Exner, H. F. Fehr, W. E. Ferguson, C. H. Fischer, Edward Fleisher, E. E. Floyd, Tomlinson Fort, J. S. Frame, Orrin Frink, J. W. Gaddum, H. M. Gehman, H. H. Germond, Wallace Givens, A. M. Gleason, H. E. Goheen, V. D. Gokhale, D. B. Goodner, E. H. Hadlock, Franklin Haimo, P. R. Halmos, P. C. Hammer, Frank Harary, F. S. Harper, E. A. Hedberg, Nicholas Heerema, M. H. Heins, Edwin Hewitt, E. H. C. Hildebrandt, A. J. Hoffman, T. C. Holyoke, A. S. Householder, J. A. Hratz, G. B. Huff, Ralph Hull, M. G. Humphreys, W. R. Hutcherson, Ernest Ikenberry, S. L. Jamison, B. W. Jones, F. B. Jones, P. S. Jones, R. V. Kadison, Shizuo Kakutani, Joseph Kampé de Fériet, H. T. Karnes, M. E. Kellar, J. L. Kelley, L. M. Kelly, A. J. Kempner, J. R. F. Kent, M. S. Klamkin, J. R. Kline, R. H. Knox, R. J. Koch, F. W. Kokomoor, Jacob Korevaar, G. B. Lang, R. E. Langer, Nathan Lazar, B. A. Lengyel, R. J. Levit, F. A. Lewis, Z. L. Loflin, Eugene Lukacs, E. J. McShane, C. C. MacDuffee, H. M. MacNeille, H. F. MacNeish, J. R. Mayor, Herman Meyer, H. A. Meyer, D. D. Miller, W. L. Miser, Benjamin Ernest Mitchell, J. M. Mitchell, C. N. Moore, L. J. Mordell, D. R. Morrison, G. D. Mostow, W. B. Moye, Zeev Nehari, John von Neumann, J. D. Newburgh, C. V. Newsom, O. M. Nikodým, A. J. Owens, O. O. Pardee, W. V. Parker, P. B. Patterson, L. G. Peck, W. D. Peeples, S. E. Pence, F. W. Perkins, I. E. Perlin, C. G. Phipps, Z. M. Pirenian, Harry Polachek, F. M. Pulliam, E. S. Quade, J. F. Randolph, L. T. Ratner, C. B. Read, L. M. Reagan, C. J. Rees, M. S. Rees, P. K. Rees, B. P. Reinsch, J. H. Roberts, F. V. Rohde, J. B. Rosser, W. A. Rutledge, Arthur Saastad, R. G. Sanger, J. W. Sawyer, M. A. Scheier, E. R. Schneckenburger, C. S. Scott, I. E. Segal, Esther Seiden, W. T. Sharp, C. N. Shuster, T. M. Simpson, C. B. Smith, W. S. Snyder, Andrew Sobczyk, J. J. Sopka, D. E. South, T. H. Southard, W. H. Spragens, H. D. Sprinkle, M. E. Stark, R. W. Stokes, P. M. Swingle, Gabor Szegö, T. T. Tanimoto, Olga Taussky, H. E. Taylor, J. M. Thomas, G. L. Tiller, John Todd, C. B. Tompkins, M. M. Torrey, E. A. Trabant, H. C. Trimble, C. A. Truesdell, A. W. Tucker, S. M. Ulam, Gilbert Ulmer, Eugene Usdin, L. A. Van Dyke, Henry Van Engen, A. H. Van Tuyl, T. L. Wade, 
R. J. Walker, A. D. Wallace, J. L. Walsh, L. E. Ward, Jr., H. F. Weinberger, Alexander Weinstein, M. J. Weiss, F. P. Welch, J. G. Wendel, G. T. Whyburn, L. S. Whyburn, W. M. Whyburn, W. L. Williams, R. L. Wilson, W. H. Wilson, G. N. Wollan, F. M. Wright, Arthur Zeichner, A. D. Ziebur.

The twenty-fourth Josiah Willard Gibbs Lecture entitled Some basic problems of statistical mechanics was delivered by Professor G. E. Uhlenbeck of the University of Michigan on Wednesday, December 27. President-elect John von Neumann presided at this lecture.

Professor J. L. Walsh of Harvard University delivered his Retiring Presidential Address entitled The location of critical points on Thursday, December 28. Professor John von Neumann, President-elect, was the presiding officer.

The Committee to Select Hour Speakers for Annual and Summer Meetings invited two speakers. On Thursday, December 28, Professor M. H. Heins of Brown University addressed the Society on Regularity of growth of subharmonic functions. President J. L. Walsh presided at this lecture. On Friday, December 29, Professor L. J. Mordell of St. John's College, Cambridge University spoke on The product of $n$ homogeneous linear forms. Professor Patrick Du Val was the presiding officer.

The annual Business Meeting and Election of Officers was held at 10:15 A.M. on Friday, December 29. Details of proceedings are reported in the sequel.

A reception for members of the mathematical organizations and their guests was given by the University of Florida following the Gibbs lecture on Wednesday evening. This was in the Social Room of the Florida Union, headquarters of the Society for the meetings.

The dinner on Friday evening in the University Cafeteria was attended by approximately 250 members and guests. Professor T. M. Simpson, Dean of the Graduate School of the University of Florida, was toastmaster. President J. Hillis Miller of the University of Florida extended greetings and Professor R. E. Langer responded on behalf of the assembled guests. President Walsh presented a sterling silver tray to Secretary Kline as a token of appreciation by Society members of Professor Kline's ten years of service as Secretary. Professor Kline followed his expression of thanks by brief mention of some of the more significant activities of the Society during his period in office. Professor P. H. Daus of the University of California, Los Angeles, offered a resolution of thanks and appreciation to the University of Florida, the Committee on Arrangements, and all who assisted in making the meetings successful and enjoyable. This resolution was adopted by a rising vote. 
The Annual Business Meeting of the Society was held on Friday morning, December 29. The Secretary reported on the affairs of the Society.

Upon recommendation of the Council, it was voted to amend the By-Laws of the Society so that the President and Treasurer will be ex officio members of the Board of Trustees.

It was voted that the members of the Society present vote to disapprove the action of the Council on the question of meetings at the University of California.

It was voted that the Secretary of the Society be instructed not to release the action of the Council concerning meetings at the University of California beyond the membership of the Society and that the Council be requested to reconsider the matter at its meeting in Minneapolis.

The Council met on Thursday evening, December 28, 1950.

The Secretary announced the election of the following thirty persons to ordinary membership in the Society:

Mr. Sholom Arzt, New York University;

Mr. Hans Boris Belck, General Electric Company, Pittsfield, Mass.;

Mr. Nicholas Bilotta, Department of Public Works, New York, N. Y.;

Visiting Professor Frank Featherstone Bonsall, Oklahoma Agricultural and Mechanical College;

Mr. Allen Virgil Butterworth, University of Chicago;

Mr. Robert Kent Butz, Alabama Polytechnic Institute;

Professor Madeleine Forró, Barat College, Lake Forest, Ill.;

Mr. Robert S. Freeman, New York University;

Mr. Thom Raymond Greene, Carnegie Institute of Technology;

Mr. Frederick Alexander Hiersch, Detroit, Mich.;

Mr. James Grady Horne, Jr., Tulane University of Louisiana;

Mr. Henry Paul Kramer, Radiation Laboratory, University of California;

Dr. Paolo Lanzano, St. Louis University;

Mr. Thomas James McNamara, Park College, Parkville, Mo.;

Professor James Horatio Means, Tillotson College, Austin, Tex.;

Mr. Gene Woodard Medlin, University of North Carolina;

Mr. Edward Peter Merkes, DePaul University;

Mr. Richard Allan Moore, Washington University;

Professor Elisha Netanyahu, Hebrew Technical College, Haifa, Israel;

Mr. John Adolph Nohel, Massachusetts Institute of Technology;

Mr. Richard Eugene Priest, University of Illinois;

Mr. Savatore John Rapisarda, Lawrence, Mass.;

Professor Arthur Saastad, DePaul University;

Mr. Federico Mariano Sioson, University of the Philippines;

Mr. H. D. Sprinkle, University of Florida;

Dr. Marie D. Wann, George Washington University;

Mr. William Hamer Warner, Carnegie Institute of Technology;

Mr. Gerard Paul Weeg, St. Ambrose College, Davenport, Iowa; 
Sister Teresa Dolores Wolf, College of Mount St. Vincent, New York, N. Y.; Mr. Ti Yen, University of Illinois.

It was reported that the following ninety-six persons had been elected to membership on nomination of institutional members as indicated:

University of Alabama: Professor Oscar Richard Ainsworth;

Brown University: Dr. George Walter Morgan;

Bryn Mawr College: Miss Padmini Rangaswamy Iyengar;

University of California: Messrs. Woodrow Wilson Bledsoe, Solomon Feferman, and Joseph William Weihe;

Cornell University: Messrs. Austin Arthur Howard and Gerson Bernhard Robison;

Duke University: Messrs. Gordon Cleaves Byers and Robert Taylor Herbst;

Equitable Life Insurance Company of Iowa: Messrs. Floyd Thomas Beasley and Tilmar Archibald Moilien;

University of Illinois: Messrs. Charles Glenn Carlyle, Alvin Vincent Fend, Joseph Hashisaki, John Edward Heisler, Jr., John William Rolley, and Robert Jerome Silverman;

Indiana University: Messrs. Jerald Laverne Ericksen, Lowell Keith Frazer, and David Eliza van Tijn;

Institute for Advanced Study: Professors Aldo Andreotti, Heinrich A. L. Behnke, Fabio Conforto, Vittorio Dalla Volta, Mr. Freeman John Dyson, Dr. István Sándor Gál, Professors Kenkichi Iwasawa, Jean Leray, Hidegorô Nakano, Giovanni Ricci, Dr. Leo Reino Sario, Professors Beniamino Segre and Kentaro Yano;

Iowa State College of Agriculture and Mechanic Arts: Mr. Arnold Marion Wedel; Johns Hopkins University: Messrs. James Richardson Belford, Harry Wilson Clarke, Enrico Thomas Federighi, Irving Elwell Gaskill, Vernon Ray Hancock, Benjamin Fulton Howard, Jr., Harold Shulman, and Charles Junior Standish;

University of Kansas: Messrs. Kuo-Chih Hsu and Arthur Herman Kruse;

Lehigh University: Mr. Jacob Samoloff;

University of Maryland: Messrs. Russell Martin Brown, Jr., Daniel Richard Hughes, and Miss Edith Moss;

Massachusetts Institute of Technology: Messrs. Richard Horace Battin and Donald William Dunn;

University of Michigan: Messrs. Richard Blaine Barrar, John Gilbert Hocking, Robert William MacDowell, and Donald Ferdinand Mela;

Ohio State University: Messrs. Richard Albert Dean, Miles Sanford Edwards, Edward Richard Fadell, and Harold M. Horwitz;

University of Oregon: Mr. Arthur Eugene Livingston;

Pennsylvania State College: Messrs. Edward Jack Cogan and Albert Russel Erskine; University of Pennsylvania: Messrs. Robert Ellis, Walter Hess Leser, and Philip Rabinowitz;

Princeton University: Messrs. Donald Wayne Bushaw, Herbert Amasa Forrester, George Allen Hufford, Roger Halliday Kyle, Marvin Lee Minsky, Peter Bjфrn Nemenyi, Onorato Timothy O'Meara, Basil Andrew Rattray, Yomei Sawanobori, and David Lee Wallace;

Purdue University: Messrs. William Richard Fuller, Louis Eugene Grosh, Ladis Daniel Kovach, Maurice Dean Lamoree, and Walter Davidson Wood;

Rice Institute: Messrs. Jim Douglas, Jr., and John Delbert Rice; 
Stanford University: Messrs. Gordon Bruce Hedrick, John Weston Hardy, Jr., Joel Franklin, and Donald Charles Benson;

Swarthmore College: Mr. Arthur Paul Mattuck;

University of Tennessee: Mr. Nickolas Heerema;

Wellesley College: Professor James Walter Warwick;

Western and Southern Life Insurance Company: Messrs. Eugene Warren Bates, Edward Bathurst Fackler, and Edwin Herbert Vonderbrink;

Yale University: Messrs. Austin Robert Brown, Jr., Stephen Peter Hoffman, Jr., Brian Eugene Owens, and Morris Weisfeld.

The Secretary announced that the person indicated below had been admitted to membership in the Society, under reciprocity agreements with various mathematical organizations: London Mathematical Society: Professor Charles Fox, McGill University.

The Secretary is pleased to report at this time that the ordinary membership of the Society is now 4411, including 353 nominees of institutional members and 47 life members. There are also 107 institutional members. The total attendance at all meetings in 1950 was 1413; the number of papers read was 436; there were 14 hour addresses, 3 half-hour addresses, 1 Gibbs Lecture, and 1 Retiring Presidential Address; the number of members attending at least one meeting was 1068.

The following appointments by the President were reported: As a Committee on Arrangements for the Summer Meeting at the University of Minnesota, September 4-7, 1951: Professors John M. H. Olmsted, Chairman, R. H. Cameron, H. M. Gehman, W. L. Hart, G. K. Kalisch, Fulton Koehler, W. S. Loud, S. E. Warschawski, and J. W. T. Youngs; as Chairman of the Committee on Printing Contracts for the period 1951-1953: Professor C. J. Rees; as Chairman of the Committee on Visiting Lectureships for the period 1951-1953: Professor R. P. Boas, Jr. (committee now consists of Professors R. P. Boas, Jr., Richard Courant, and H. S. Wall); as a member of the Committee on Places of Meetings for the period 1951-1953: Professor Orrin Frink (committee now consists of Professors R. W. Brink, Chairman, Orrin Frink, and J. M. Thomas); as members of the Committee on Applied Mathematics for the period 1951-1953: Professors R. V. Churchill and M. H. Martin (committee now consists of Professors M. H. Martin, Chairman, R. V. Churchill, K. O. Friedrichs, John von Neumann, William Prager, and Eric Reissner); as tellers for the 1950 annual election: Professors L. W. Cohen, F. W. Kokomoor, and E. R. Kolchin; as a member of the Committee to Select Hour Speakers for Summer and Annual Meetings for the period 19511952: Professor Deane Montgomery (committee now consists of Professors E. G. Begle, Chairman, A. A. Albert, and Deane Mont- 
gomery); as members of the Committee to Select Hour Speakers for Eastern Sectional Meetings: Professor N. E. Steenrod (to replace Professor E. G. Begle, term to expire December 31, 1951) and Professor M. H. Heins for the period 1951-1952 (committee now consists of Professors L. W. Cohen, Chairman, M. H. Heins, and N. E. Steenrod); as a member of the Committee to Select Hour Speakers for Southeastern Sectional Meetings for the period 1951-1952: Professor B. J. Pettis (committee now consists of Professors W. M. Whyburn, Chairman, Tomlinson Fort, and B. J. Pettis); as a member of the Committee to Select Hour Speakers for Western Sectional Meetings for the period 1951-1952: Professor P. R. Halmos (committee now consists of Professors J. W. T. Youngs, Chairman, P. R. Halmos, and Walter Leighton); as a member of the Committee to Select Hour Speakers for Far Western Sectional Meetings for the period 19511952: Professor A. P. Morse (committee now consists of Professors J. W. Green, Chairman, M. R. Hestenes, and A. P. Morse); as Editor of Volume IV of the Proceedings of Symposia on Applied Mathematics: Professor M. H. Martin; as Chairman of the Committee to Consider Non-editorial Problems of the Memoirs: Professor A. W. Tucker.

It was reported that Professor W. T. Reid's name was substituted for Professor E. G. Begle as a nominee for membership at large of the Council on the ballot for the annual election.

At the annual election which closed on December 28, 1950, and at which 575 votes were cast, the following officers were elected:

Vice President, Professor G. A. Hedlund.

Secretary, Professor E. G. Begle.

Associate Secretary, Professor L. W. Cohen.

Treasurer, Dean A. E. Meder, Jr.

Librarian, Professor Jekuthiel Ginsburg.

Member of the Editorial Committee of the Bulletin, Professor W. T. Martin.

Member of the Editorial Committee of the Proceedings, Professor Nathan Jacobson.

Member of the Editorial Committee of the Transactions, Professor G. T. Whyburn.

Member of the Editorial Committee of the Colloquium Publications, Professor A. A. Albert.

Member of the Editorial Committee of Mathematical Reviews, Professor William Feller.

Member of the Editorial Committee of Mathematical Surveys, Professor Leo Zippin. 
Representative on the Editorial Board of the American Journal of Mathematics, Professor Reinhold Baer.

Members at large of the Council, Professors D. H. Blackwell, H. F. Bohnenblust, H. S. M. Coxeter, Irving Kaplansky, and W. T. Reid. Members of the Board of Trustees, Professors B. P. Gill, T. H. Hildebrandt, and J. R. Kline.

The Council voted unanimously to adopt the following three resolutions:

Whereas Professor John Robert Kline assumed the office of Secretary of the American Mathematical Society in 1941 and is now relinguishing that office after ten years of faithful and devoted service, and

Whereas Professor Kline has performed the duties of his office with such distinction that the Society has functioned in an outstanding manner and has enjoyed most wholesome growth, and

Whereas in discharging the heavy and important work of the Secretaryship Professor Kline has always given his time and physical energy generously and without stint, and

Whereas it is the wish of the Council of the American Mathematical Society to record its deep gratitude to Professor Kline:

$\mathrm{Be}$ it therefore resolved that the Council hereby express its most profound thanks to Professor Kline for his contributions to the development of mathematics in America through his work in the office of Secretary of the American Mathematical Society and otherwise, and

Be it further resolved that copies of this resolution be sent to Professor Kline, spread upon the minutes of the Council, and appropriately published by the Society.

The Council of the American Mathematical Society records its deep gratitude to Professor Arnold Dresden for the outstanding service which he has rendered through the office of Librarian for the Society. Under his able and careful handling, the Society library has grown steadily to a point where it now represents a highly valuable asset, both as to content and as to financial value. Through carefully selected and arranged exchanges, Professor Dresden has acquired much valuable material without drain on the funds of the Society. Through cataloging, arrangement, and numerous other services, he has increased the usefulness of the library to the Society membership.

The Council hereby expresses its appreciation to Professor Dresden and conveys to him its most profound thanks for the unselfish devotion to the service of American mathematics which he has ever shown in the performance of his duties as Librarian of the Society.

The Council of the American Mathematical Society records its deep gratitude to Professor Temple Rice Hollcroft for contributions which he has made to American mathematics through his many years of devoted and efficient service as Associate Secretary of the Society. Under his careful handling of the duties of his office, meetings of the Society for which he carried responsibility have been conducted with the smoothness that reflects careful thought and good planning. Through his programming, conducting, and reporting Society meetings, and through the multitude of other services encumbent upon his office, he has contributed greatly to the success of activities of the Society.

The Council hereby expresses its appreciation to Professor Hollcroft and conveys 
to him its most profound thanks for the fine service which he has rendered the American Mathematical Society through his work as Associate Secretary.

The President was authorized to appoint a committee to consider the request of Mrs. Charles C. Torrance that wives of members of the Society who are mathematicians be given the same privileges as emeritus members.

The Council voted that the following resolution be adopted:

Owing to the expressed reluctance of a large number of mathematicians to attend meetings of the American Mathematical Society at the University of California as long as the conditions previously condemned prevail, the Society will not hold meetings on a campus of the University of California until these conditions are alleviated.

It was voted that a copy of the resolution be sent to the editors of Science.

The Council voted to approve the following dates of meetings of the Society in 1951: November 23-24, at the University of Oklahoma; December 26-28 in Providence, Rhode Island.

In an appendix to this report are excerpts from the report of the Treasurer for the fiscal year 1950 as verified by the auditors. A copy of the complete report will be sent, on request, to any member of the Society.

The Librarian reported the following additions to the Library: 163 bound volumes of periodicals, 80 books, and 46 pamphlets.

Certain invitations to give addresses in 1951 were announced: Professors Zeev Nehari and Tadasi Nakayama for the February meeting in Cincinnati.

Upon recommendation of the Committee to Nominate a Representative of the Society on the Policy Committee, Professor J. R. Kline was elected to serve on the Policy Committee for a four-year term beginning January 1, 1951.

The Bulletin Editorial Committee reported that the journal is more nearly current in its publication schedule than has been true for some years. A recommendation that a total of 600 pages be authorized for the 1951 Bulletin was accepted by the Council and referred to the Trustees. Professor R. P. Boas was reported as a new Assistant Editor for the Bulletin.

The Transactions and Memoirs Editorial Committee reported that the Transactions will have published almost exactly the 1100 pages authorized for 1950. The interval between receipt of the manuscripts and publication usually falls between six and nine months. The Council voted to recommend to the Board of Trustees that 1100 pages be published in the Transactions in 1951. In the Memoirs series, Number 1 appeared in July and Number 2 is now being 
printed. Four additional numbers are ready for the printer and it is hoped that they will be available early in 1951 .

The Mathematical Reviews Editorial Committee reported that 766 pages of reviews had been published, the same as reported for 1949. The subscription list as of November, 1950, was 2,061. Beginning September 1, Dr. R. P. Boas, Jr. was replaced by Dr. J. V. Wehausen as Executive Editor.

The Proceedings Editorial Committee reported that the backlog of the journal was such that a period of approximately fourteen to sixteen months must now elapse between acceptance and publication of a particular paper. A recommendation that 1000 pages be authorized for the 1951 Proceedings was accepted by the Council and referred to the Trustees. This represents an increase of 150 pages over the 1950 volume of the Proceedings. Professors P. T. Bateman, N. J. Fine, A. W. Goodman, and E. H. Spanier were reported as new Assistant Editors for the Proceedings.

The Organizing Committee of the International Congress reported that the International Congress held at Harvard University from August 30 through September 6, 1950, was a complete success. The next International Congress of Mathematicians will be held in Amsterdam in 1954. Over 2,300 persons registered for the Congress, of whom about 300 came from outside of the continental United States and Canada. The Proceedings of the Congress, which represent the only unfinished business of the Congress, are expected to appear in 1951; they will comprise two volumes of about 850 pages each. In addition, the Conference on Algebraic Tendencies in Analysis will be published as a separate volume in the Mathematical Surveys Series, provided the manuscripts are received before March $1,1951$.

The Committee on Reciprocity Agreements reported that, "While it is felt that the American Mathematical Society should not enter into reciporicity agreements of the usual sort with Societies in the American area, it is strongly recommended that, as occasion permits, mathematicians residing in contiguous territory to the United States be extended cordial invitations to become members of the A.M.S. and that these invitations be supplemented by offering a favorable exchange rate where possible." The Council voted to accept the report and to discharge the Committee.

Upon recommendation of the Policy Committee, the Council voted that the National Council of Teachers of Mathematics be asked to designate one representative to serve on the Policy Committee. 
The Colloquium Committee recommended that Professor Alfred Tarski be invited to deliver the Colloquium Lectures at the 1952 Summer Meeting. The Council voted to accept this recommendation.

The Council voted to adopt the following resolution:

Since its founding sixteen years ago, the Duke Mathematical Journal has formed an important integral part in the American Mathematical Society's program for the publication of mathematical research. The value of the aid thus furnished by the Duke University has been greatly enhanced by the fact that the Society has not been called upon to share the financial burden. This assistance is especially significant in this period of heavy publication needs and strained Society resources.

The officers and members of the Society deeply appreciate the nature and the extent of Duke's contribution. The Council conveys the gratitude of the Society and expresses its sincere hope that Duke University will maintain the Journal at the prewar levels of size and quality.

The Council voted to recommend to the Board of Trustees that a joint committee of the Council and the Board of Trustees be appointed to be available for consultation on matters relating to the Duke Mathematical Journal.

Abstracts of papers read follow. Presiding officers at the sessions for contributed papers were Professors P. H. Daus, C. R. Adams, W. V. Parker, J. B. Rosser, Tomlinson Fort, W. L. Duren, A. W. Tucker, Dr. Mina S. Rees, and Dr. A. S. Householder.

Papers whose abstract numbers are followed by the letter " $t$ " were read by title. Paper number 101 was presented by Mr. Zeichner, 103 by Professor Bonsall, 112 by Professor Sobczyk, 115 by Professor Hewitt, and 138 by Dr. Weinberger. Dr. Amitsur was introduced by Dr. Jakob Levitzky, Dr. Dekker by Professor P. C. Rosenbloom, Mr. Peters by Professor Tomlinson Fort, and Professor Arf by Professor Alexander Weinstein.

\section{Algebra AND THEORY OF NUMBERS}

\section{0t. S. A. Amitsur: Radical-like ideals.}

In the present paper an axiomatic study of the various radical-like ideals is initiated. This is achieved by considering properties $E$ of rings which satisfy the following conditions: (1) The zero ring is an $E$-ring, that is, a ring which possesses the property $E$. (2) Each homomorphic image of an $E$-ring is also an $E$-ring. (3) If $S$ is not an $E$-ring, then $S$ contains a proper radical $E$-ideal, that is, an ideal $Q$ such that the nonzero quotient ring $S / Q$ contains no ideals with the property $E$ ( $E$-ideals), except the zero ideal. (4) Every ideal in an $E$-ring is an $E$-ideal. (5) If $R$ is a right $E$-ideal in $S$, then $S R$ is also an $E$-ideal in $S$. Some of the results obtained are: (I) If $E$ satisfies (1) and (2), then the intersection of all the radical $E$-ideals of $S$ is also a radical $E$-ideal (the lower $E$-radical). (II) If $E$ satisfies the three conditions (1)-(3), then the lower $E$-radical of $S$ is the maximal $E$-ideal in $S$ (the $E$-radical of $S$ ). (III) if $E$ satisfies (1)-(4), then the $E$-radical of an ideal $A$ in $S$ is the intersection of $A$ and the $E$-radical of $S$. (IV) If $E$ satisfies all conditions (1)-(5), then the radical $N$ of $S$ contains all 
right $E$-ideals of $S$ and $S / N$ contains no right $E$-ideals except the zero. (Received November 17, 1950.)

\section{Eckford Cohen: Sums of products in $G F\left[p^{n}, x\right]$.}

In a previous paper ( $A n$ extension of Ramanujan's sum, Duke Math. J. vol. 15 (1948) pp. 85-90), the author deduced the number of representations of a polynomial $F$ in $G F\left[p^{n}, x\right]$ as a sum of products, $F=\sum_{1}^{r} \alpha_{i} X_{i}^{e} Y_{i}$, with constant coefficients $\alpha_{i}$, the $X_{i}, Y_{i}$ satisfying certain conditions on degree. In the present paper, it is shown that certain types of polynomial coefficients can be introduced without materially altering the previous results. The methods used involve the evaluation of arithmetic sums which arise in connection with other problems as well. (Received November 17, 1950.)

\section{2t. Harvey Cohn: On stable maxima for the product of homogeneous} forms. II.

Continuing from a previous abstract (Bull. Amer. Math. Soc. Abstract 57-1-15), the author applies to his definitions of stable extrema the forms $|N(\theta) / D|$ where $N(\theta)$ and $D$ are the norm and determinant in an algebraic module of degree $n$. Let $q=n(n-1)+1$ algebraic numbers $\Phi^{(k)}$ of minimum norm be given, with the $q$ vectors, $\Phi_{i}^{(k)} / \Phi_{j}^{(k)}$ (where the $n(n-1)$ components $i, j$ with $i \neq j$ refer to conjugates). Then consider sets of $q$ integers $A_{k}$ such that $\sum A_{k} \Phi_{i}^{(k)} / \Phi_{j}^{(k)}=0$. If such $A_{k}$ are all of the same sign, or all zero, then the form has a stable extremum. Thus a differential property is decidable within the normal field. It is then seen that for the absolute extrema (critical lattices) solved by Davenport $\left(n=3, D^{2}=49\right.$ and $\left.D^{2}=-23\right)$, the critical lattices for the product provide a stable minimum in the sense of the author's definitions. (Received November 3, 1950.)

83. J. C. E. Dekker: The constructivity of maximal sum ideals in a certain Boolean algebra.

A collection of positive integers will be called a set; a collection of sets will be called a class. If $V$ is the class of all sets, $V$ forms a Boolean algebra with respect to the operations of union and intersection. One classifies the maximal sum ideals in a Boolean algebra as atomic or nonatomic. It is generally suspected that while the atomic maximal sum ideals are constructive, the nonatomic maximal sum ideals are not constructive. The nature of this conjecture is however not clear, since no definition of a constructive sum ideal in a Boolean algebra is generally accepted. In this paper the concept of a recursively enumerable class is introduced. This concept enables one to propose two definitions for a constructive sum ideal in the Boolean algebra $V$. It is shown that according to each of these definitions, a maximal sum ideal in $V$ is constructive if and only if it is atomic. The constructivity of several types of sum ideals in $V$ is investigated. It is shown that the two definitions which are proposed for a constructive sum ideal in $V$ are not equivalent. (Received November 6, 1950.)

\section{J. C. Eaves: A set of matrices possessing the "p" property.} Preliminary report.

In his paper On k-commutative matrices [Trans. Amer. Math. Soc. vol. 39 (1936) pp. 483-495] W. E. Roth introduced $k$-commutative matrices and obtained, among other properties, the skeleton of a matrix $B=\left(B_{i j}\right)$ such that $A=\left(A_{i i}\right)$ is $k$-commutative with respect to $B$, where $A$ is taken to be a Jordan canonical matrix. Later 
[On the characteristic values of the matrix $f(A, B)$, Trans. Amer. Math. Soc. vol. 39 (1936) pp. 234-243] Roth discussed the skeletons of a pair of matrices, $A$ and $B$, which have property " $p$." These papers suggest a set of block matrices $R_{\lambda}=\left(R_{\lambda i j}\right)$ $(\lambda=1,2, \cdots, k)$ where $R_{\lambda i j}$ is an $n_{i} \times n_{j}$ matrix $\left(n_{i}=n_{j}\right.$ for, $i=j ; n_{s} \neq n_{t}$ where $s \neq t$ for $R_{s s}, R_{t t}$ ) with the first $\left\{n_{i}, n_{j}\right\}-1$ diagonals of each $R_{\lambda i j}$ containing only zero elements. There exists a product, $P$, of elementary permutative matrices such that for any $R_{\lambda}$ then $P^{-1} R_{\lambda} P=T_{\lambda}$ where $T_{\lambda}$ is a triangle matrix. This result is a generalization of Roth's second paper mentioned above. The matrices $R_{\lambda}(\lambda=1,2, \cdots, k)$ possess the triangle property and the " $p$ " property and are $k$-commutative in pairs. This result is not necessarily true in case $n_{s}=n_{t}, s \neq t$ for $R_{s s}, R_{t t}$, even though several other restrictions are placed upon the $R_{\lambda}$. (Received November 13,1950.)

\section{5t. D. O. Ellis: Notes on the foundations of lattice theory}

A lattice may be defined as a system closed under two binary single-valued operations, $a \bigvee b$ and $a \wedge b$, which satisfies: 1 . Double commutativity $(a \bigvee b=b \bigvee a$ and $a \wedge b$ $=b \wedge a)$; 2. Double associativity $(a \bigvee(b \bigvee c)=(a \bigvee b) \bigvee c$ and $a \wedge(b \wedge c)=(a \wedge b) \wedge c)$; 3. Double idempotency $(a \bigvee a=a \wedge a=a)$; and 4. Alternation $(a \bigvee b=a$ if and only if $a \wedge b=b)$. Systems satisfying 1,2 , and 4 are called pseudo-lattices. Systems satisfying $1,2,3,4$, and 5. Single distributivity (either $a \bigvee(b \wedge c)=(a \bigvee b) \wedge(a \bigvee c)$ for all $a, b, c$ or $a \wedge(b \bigvee c)=(a \wedge b) \bigvee(a \wedge c)$ for all $a, b, c)$ are called distributive lattices. For pseudolattices, it does not follow that each identity of 5 implies the other so that a pseudolattice satisfying 6. Double distributivity (both identities of 5) is called a distributive pseudo-lattice. An element $a$ of a pseudo-lattice is called finitely potent if it satisfies 7. $\exists n$ (a natural integer) $\ni \bigwedge_{n+1} a=a$ or $\bigvee_{n+1} a=a$; where $\bigwedge_{n+1} a$ and $\bigvee_{n+1} a$ indicate "powers" or "multiples" of $a$. In this note it is shown that elements satisfying 7 in a system $(1,2,4,6)$ satisfy 3 and, hence, that a distributive pseudo-lattice each of whose elements is finitely potent is a distributive lattice. In nondistributive pseudo-lattices, 7 does not imply 3. (Received July 24, 1950.)

\section{J. S. Frame: An irreducible representation extracted from two permutation groups.}

Let $G$ be a finite group of order $O_{G}$, containing two subgroups $A$ and $B$, of indices $m$ and $n$ respectively, such that the double coset $A B$ contains $p O_{G}$ elements, and all the remaining $q \mathrm{O}_{G}$ elements of $G$ belong to a second double coset $A g_{2} B$. The subgroups $A$ and $B$ induce representations of $G$ by permutation matrices $R^{A}(g)$ and $R^{B}(g)$ of degrees $m$ and $n$ respectively, having as common irreducible components just two irreducible representations: $F_{1}$, the identity representation, and $F_{2}$, which is to be determined. An $m \times n$ incidence matrix $V=\left(v_{i j}\right)$ is defined by setting $v_{i j}=1$ or 0 , according as the right cosets $A \rho_{i}$ and $B \sigma_{j}$ have or have not a common element, and an $m \times m$ correlation matrix $K$ is formed from the rows of $V$, having $k^{2}$ as the mean square of its elements. It is proved that the degree $f$ of $F_{2}$ is $1 / k^{2}$, and the trace $\chi_{\lambda}$ of the matrices of $F_{2}$ for the $h_{\lambda}$ elements of a class $C_{\lambda}$ is $\chi_{\lambda}=f\left(1-q_{\lambda} / q\right)$, where $q_{\lambda} h_{\lambda}$ denotes the number of elements of $C_{\lambda}$ not in $A B$. Finally, a right ideal basis for $F_{2}$ is obtained from the ring elements $\vec{A} \bar{B} \sigma_{j}-\bar{G}$, where $\bar{A}, \bar{B}$, and $\bar{G}$ denote averages of the elements of $A, B$, or $G$ in the group ring. (Received October 30,1950.)

\section{V. D. Gokhale: Concerning the determinant of a Hermitian matrix of order 3 with elements in Cayley algebra.}

After defining the determinant for a Hermitian matrix, the paper proves that the 
determinant is unchanged by simultaneous interchanges of rows and columns, and that the determinant of the square of a matrix is equal to the square of the determinant of the matrix. An adjoint matrix is defined and it is shown that the product of a matrix and its adjoint is a diagonal matrix whose elements, in case the elements of the original matrix associate with each other, are the value of the determinant of the original matrix. (Received November 8, 1950.)

88. E. H. Hadlock: $A$ method of construction of an improperly primitive form.

A method is derived and stated whereby, given the integral invariants $\Omega, \Delta$ and possible sets of progressions involving 2 and the odd prime factors of $\Omega$ and $\Delta$, the integral coefficients $a, b, c, r$, and $s$ are determined in the improperly primitive form $f=a x^{2}+b y^{2}+c z^{2}+2 r y z+2 s x z$ with determinant $d=\Omega^{2} \Delta \neq 0$ so that $f$ will have associated with it the given invariants and sets of progressions. (Received November 6, 1950.)

89. Franklin Haimo: Dense ideals in Boolean algebras. Preliminary report.

Those ideals in Boolean algebras are studied which are the image spaces of endomorphisms $\alpha$ with the property $\alpha(x) \leqq x$. (Received November 13,1950.)

90. T. C. Holyoke: On the structure of multiply transitive permutation groups.

Given a finite permutation group $G$ fixing the letter 0 and transitive on the remaining letters $1, \cdots, n$, several sets of necessary and sufficient conditions are determined for the existence of a group $H$ which is transitive on letters $0,1, \cdots, n$ and contains $G$ as the (largest) subgroup fixing the letter 0 . Considering $G$ and $H$ to have an additional fixed letter, it is shown that the number of distinct groups $H$ in which the given group $G$ can be so embedded is greater than the number of distinct groups in which any one of the groups $H$ can be similarly embedded. It is further shown that, once all groups which are exactly doubly transitive have been determined, the problem of constructing all groups with higher degree of transitivity is reduced to a very simple, straightforward process. New restrictions on the structure of $k$-fold transitive groups are found in terms of certain normalizers of the subgroups fixing $1,2, \cdots, k$ given letters. (Received November 13, 1950.)

91t. L. K. Hua and Irving Reiner: Automorphisms of the unimodular group.

Let $\mathfrak{M}_{n}$ denote the group of $n$-rowed unimodular matrices, and let $\mathfrak{A}_{n}$ be the group of all automorphisms of $\mathfrak{M}_{n}$. For $n \neq 2$, it is shown that $\mathfrak{A}_{n}$ is generated by the set of all inner automorphisms of $\mathfrak{M}_{n}$, the automorphism $X \in \mathfrak{M}_{n} \rightarrow\left(X^{\prime}\right)^{-1}$, and (for even $n$ only) the automorphism $X \in \mathfrak{M}_{n} \rightarrow(\operatorname{det} X) \cdot X$. It is also proved that $\mathfrak{A}_{2}$ is generated by the above and an additional automorphism $\tau$ given as follows: for $X \in \mathfrak{M}_{2}$ and det $X=1$, set $\epsilon(X)=1$ or -1 according as $X$ is or is not an element of the commutator subgroup of $\mathfrak{M}_{2}$, and let $J$ be a fixed element of $\mathfrak{M}_{2}$ with $\operatorname{det} J=-1$; then define $X^{\tau}=\epsilon(X) \cdot X$ for $\operatorname{det} X=1, X^{\tau}=\epsilon(J X) \cdot X$ for $\operatorname{det} X=-1$. The proof is based on the establishment of canonical forms for involutions under similarity transformations, and normalizer subgroups of such canonical forms. (Received November 17, 1950.) 


\section{B. W. Jones: On automorphs of symmetric matrices of odd order.}

Let $A$ be a symmetric matrix of odd order and $S$ an arbitrary skew matrix of the same order. Automorphs of $A$ are expressed as polynomials in $S A$ whose coefficients are explicitly given functions of the coefficients of the characteristic function of $S A$. The latter coefficients are shown to be forms in the elements of $S$ whose matrices are concomitant matrices of $A$. These results have some advantages over Cayley's form. The method is partially based upon that of Cantor and Bachman for ternary forms. (Received November 9, 1950.)

\section{3t. Jakob Levitzki: On rings with bounded index.}

Let $R$ be a ring satisfying the following conditions: (1) Every non-nil right ideal contains a nonzero idempotent. (2) the indices of the nilpotent elements of $R$ are bounded (with bound $=n$ ). It is shown that if $R$ is semi-simple (in the sense of Jacobson) and $A \supset 0$ is an ideal in $R$, then there exists an ideal $B$ with unit such that $A \supseteq B$, $B \cong C_{m}, m \leqq n$, where $C_{m}$ is a ring of all $m$ by $m$ matrices over a strongly regular ring $C$ (that is, $C$ satisfies condition (1) and has no nilpotent elements). If $R$ is semi-simple it follows that $R$ is isomorphic with a subdirect sum of rings of type $C_{m}$. If in particular $R$ is simple, then $R \cong C_{m}$, where $C$ is a field. This generalizes a theorem of Jacobson (Ann. of Math. vol. 46 (1945) Theorem 4). If $R$ is semi-simple and the minimal (maximal) condition for two-sided ideals does not hold, then $R$ contains an infinite number of central idempotents. This generalizes a result of Kaplansky (Trans. Amer. Math. Soc. vol. 68 (1950) p. 70). A characteristic minimal (maximal) condition for two-sided ideals is derived for a ring satisfying (1) and (2) to be semi-primary in the Wedderburn-Artin sense. In particular: If $R$ is a simple nilring with bounded index, then $R$ is a nilpotent null-ring. This answers a question raised by Jacobson (loc. cit. p. 700). (Received November 17, 1950.)

\section{4t. L. H. Loomis: Free $\sigma$-complete Boolean alegbras.}

Let $m$ be any cardinal number. It is shown that there exists a $\sigma$-complete Boolean algebra $B$ with a generating set of cardinal number $m$ such that any other $\sigma$-complete Boolean algebra with a generating set of cardinal number $m$ is a $\sigma$-homomorphic image of $B . B$ is necessarily unique, and can be realized as a $\sigma$-algebra. The crux of the proof consists of showing that if $b$ is a nonzero element of any generated Boolean $\sigma$-algebra than there exists a sequence $\left\{b_{n}\right\}$, where each element $b_{n}$ is either a generator or the complement of a generator, such that $\bigcap_{1}^{\infty} b_{n} C b$. (Received November 22, 1950.)

\section{5t. G. D. Mostow: On an assertion of $A$. Weil.}

A locally compact group is said to have property (1) if there is some compact neighborhood of the identity invariant under all inner automorphisms; property (2) if there are arbitrarily small neighborhoods of the identity invariant under inner automorphisms. In his book, Integration des les groupes topologique, p. 129, Weil asserts that (2) is a consequence of (1). This assertion is proved false, even for Lie groups, by a counter example. However, the theorem is almost true for Lie groups in the following sense. Theorem. Let $G$ be a Lie group with property (1). Then there is a compact connected central subgroup $C$ such that $G / C$ is the direct product of compact and abelian subgroups and thus satisfies property (2). Thus (a) the universal covering group of $G$ is the direct product of a compact subgroup and a nilpotent subgroup, (b) the commutator subgroup of the radical of $G$ is in $C$, (c) if $G$ is 
simply connected, property (1) implies property (2), (d) if a neighborhood of the identity of $G$ is separated by finite-dimensional representations of $G$, then property (1) implies property (2). (Received September 18, 1950.)

96. W. A. Rutledge: The Hurwitz integral quaternions as a principal ideal domain.

Let $J$ represent the class of Hurwitz integral quaternions $a=a_{1} \rho+a_{2} i+a_{3} j+a_{4} k$ in which $a_{1}, a_{2}, a_{3}$, and $a_{4}$ are rational integers and $\rho=(1+i+j+k) / 2$. Then $J$ is a principal ideal domain in which the modules $J-a J, J-b J$ are $J$-isomorphic if and only if $a$ and $b$ are similar in the sense of Ore. Writing $a$, and likewise $b$, in a unique standard form $a=2{ }^{f} r(1+i)^{e} c$ where $r$ is a rational odd integer, $e$ is zero or one, $c$ is a quaternion without rational integral factor and of odd norm, the general theory of principal ideal domains is applied to $J$ to get the following. (1) If $J a J \subseteq J c \cap c J$ we say $c$ is a total divisor of $a$. Then $a$ and $b$ have the same total divisors if and only if they have the same rational factors and same exponents of $(1+i)$. (2) Every ideal $a J$ is bounded and the bound is generated by $a^{*}=2^{f} r N(c) \cdot(1+i)^{e}$ where $a=2^{f} r(1+i)^{e} c$, $N(c)$ being the norm of $c$. (3) The module $J-a J$ is indecomposable if and only if either $a$ has no rational integral factor and $N(a)$ is a prime power or else $N(a)=2^{m}$. (4) Two integral quaternions $a$ and $b$ are similar if and only if they have the same norm and bound. (Received November 13, 1950.)

97t. Olga Taussky and John Todd: The characteristic roots of products of matrices with rational integral elements.

Let $A$ and $B$ be square matrices with rational integral elements and irreducible characteristic equation. The algebraic number field in which the characteristic roots of $A B$ lie is investigated, in particular in the case that $B=A^{\prime}$, the transpose of $A$. In exceptional cases the field can be identical with the field of the roots of $A$. Not every field can, of course, occur. Quadratic fields are studied in detail. (Received November 13, 1950.)

\section{T. L. Wade: Conjugate tensors.}

Let $T_{i_{1} i_{2} \cdots i_{p}}$ be an arbitrary covariant tensor (that is, one in which the $p$ indices satisfy no particular type of symmetry), and let $T_{i_{1} i_{2} \cdots i_{p}}=\sum_{[\alpha]} T_{i_{1} i_{2} \cdots i_{p}}$ represent the decomposition of $T_{i_{1} i_{2}} \cdots i_{i_{p}}$ into tensors of various symmetry types. For each invariant type of symmetry $[\alpha]$ there is an absolute numerical tensor ${ }_{[\alpha]} I_{j_{1} j_{2} \ldots j_{p}}^{i_{1} i_{2} \ldots j_{p}}$ $\equiv_{[\alpha]} I_{(j)}^{(i)}$, called an immanant tensor, and each immanant tensor has a rank tensor ${ }_{[\alpha]} I_{(j 1)(j 2) \ldots\left(i_{r}\right)}^{\left(i_{1}\right)\left(i_{i}\right) \ldots\left(i_{r}\right)}$. For any $[\alpha]$ the rank tensor factors into two split tensors ${ }_{[\alpha]} I^{\left(i_{1}\right)\left(i_{2}\right) \cdots\left(i_{r}\right)}$ and ${ }_{[\alpha]} I_{\left(i_{1}\right)\left(i_{2}\right) \cdots\left(i_{r}\right)}$. With the aid of these split tensors the familiar concept of the conjugate tensor of a skew symmetric tensor (see Maria Pastori, Tensori emisimmetrici coniugati, Atti della Accademia Nazionale dei Lincei. Rendiconti (6) vol. 16 (1932) pp. 216-220) is generalized to give the conjugate tensor of a tensor ${ }_{[\alpha]} T_{i_{1} i_{2}} \cdots i p$ of any invariant type of symmetry, and these general conjugate tensors are studied. (Received November 13, 1950.)

\section{ANALYsis}

99t. H. A. Antosiewicz: On characteristic exponents and reduction theorems for linear differential systems.

Let $d x / d t=A(t) x$ be a linear system of differential equations where the elements 
$a_{i j}(t)(i, j=1,2, \cdots, n)$ of the matrix $A(t)$ are real, continuous functions for all $t \geqq 0$ for which there exist constants $\alpha_{i j}$ such that (I) $\lim _{t \rightarrow \infty} t^{-1} \int_{0}^{t} a_{i j}(\tau) d \tau=\alpha_{i j}$, (II) $\int_{0}^{t}\left(a_{i j}(t)-\alpha_{i j}\right) d \tau=O(1)$ as $t \rightarrow \infty$. The following special cases are considered: (i) the integrals $\int_{0}^{\infty}\left(a_{i j}(\tau)-\alpha_{i j}\right) d \tau$ converge absolutely, (ii) the functions $a_{i j}(t)$, in addition to satisfying (I) and (II), are monotonic increasing for sufficiently large $t$. In each case it is shown that the characteristic exponents of the system considered are uniformly bounded. If the characteristic roots $\rho_{1}, \rho_{2}, \cdots, \rho_{n}$ of the matrix $\left(\alpha_{i j}\right)$ are real and distinct, there exists a base $x^{1}, x^{2}, \cdots, x^{n}$ of solutions for which $\lim _{t \rightarrow \infty} t^{-1} \log \left\|x^{i}\right\|$ $=\rho_{i}$ and $\log \left\|x^{i}\right\|-\rho_{i} t=O(1)$ as $t \rightarrow \infty$. This base is used to construct a matrix $C(t)$, $C(t)$ and $C^{-1}(t)$ having bounded elements, such that if $y=C^{-1}(t) x$, the system considered is transformed into $d y / d t=B(t) y$ where the matrix $B(t)$ is diagonal. This verifies in part a conjecture by S. P. Diliberto (On systems of ordinary differential equations, Annals of Mathematics Studies, no. 20, Princeton University Press, 1950), and represents a first generalization of the classical representation theorems for linear differential systems with constant or periodic coefficients. (Received November 10, 1950.)

\section{Nachman Aronszajn: Construction of reproducing kernels and} Green's functions.

A general method for the construction of reproducing kernels is described which is based on the classical C. Neumann's series for the inverse of an operator. The method is then applied to the construction of Green's functions. It allows the construction of the Green's function for a differential problem if the Green's function for another suitable problem is known explicitly. (Received November 13,1950.)

\section{Nachman Aronszajn and Arthur Zeichner: $A$ new type of} auxiliary problem for approximation of eigenvalues by Weinstein's method.

A new type of auxiliary problem for the approximation of eigenvalues by Weinstein's method is introduced. This new type allows the application of the method in many cases when the two previously known types (see N. Aronszajn, Proc. Nat. Acad. Sci. U.S.A. vol. 34 (1948) pp. 474-480, 594-601) cannot be applied. The new auxiliary problem is obtained by relaxation of continuity conditions inside the given domain. This procedure is particularly useful for those domains which are composed of a finite number of domains (with only boundary points in common), in each of which the problem is solvable. (Received November 13, 1950.)

102t. Lipman Bers: Partial differential equations and generalized analytic functions. II. Preliminary report.

Let $F(z), G(z), z=x+i y$, be a pair of functions such that (i) $\operatorname{Im}(\bar{F} G)>0$, (ii) $F_{x}, F_{y}, G_{x}, G_{y}$ exist and satisfy a Holder condition. Every complex-valued function $w(z)$ admits the unique representation $w=\phi F+\psi G ; \phi, \psi$ real. $w$ is called $(F, G)$-pseudoanalytic in $D$ if at all points $z_{0}$ of $D$ there exists the $(F, G)$-derivative $w\left(z_{0}\right)$ $=\left\{d\left[w(z)-\phi\left(z_{0}\right) F(z)-\psi\left(z_{0}\right) G(z)\right] / d z\right\}_{z=z_{0}}$. This definition leads to a theory which (1) reduces to classical function theory for $(F, G)=(1, i)$, (2) generalizes most of the fundamental concepts and results of classical function theory, (3) bears the same relationship to the general theory of second order linear homogeneous partial differential equations of elliptic type as classical function theory does to that of the Laplace equation, (4) contains previous results on generalized analytic functions (L. Bers and A. 
Gelbart, Trans. Amer. Math. Soc. vol. 56 (1944) pp. 67-93; L. Bers, Proc. Nat. Acad. Sci. U.S.A. vol. 36 (1950) pp. 130-136) as special cases. (Received September 21, 1950.)

103. F. F. Bonsall and Morris Marden: Zeros of self-inversive polynomials.

Given the polynomial $f(z)=\sum_{0}^{n} a_{k} z^{k}$, let $f^{*}(z)=z^{n} \bar{f}(1 / z)=\sum_{0}^{n} \bar{a}_{k} z^{n-k}$. The SchurCohn method of enumerating the zeros of $f(z)$ within the unit circle $|z|=1$ depends, in the case $\left|a_{0}\right| \neq\left|a_{n}\right|$, upon applying Rouche's Theorem to the polynomial $f_{1}(z)$ $=\bar{a}_{0} f(z)-a_{n} f^{*}(z)$ or to $f_{1}^{*}(z)$. In the case, however, that $f(z)$ is self-inversive, that is, if $f(z)=u f^{*}(z),|u|=1$, the method requires the use of the Cohn theorem that $f(z)$ has the same number of zeros in the unit circle as does $\left[f^{\prime}(z)\right]^{*}$. As proved by Cohn, this theorem appears to be unrelated to the theorem involving $f_{1}(z)$. In the present note, the authors give a simpler and more natural proof of the Cohn theorem based upon their observation that for any self-inversive polynomial $f(z),\left[f^{\prime}(z)\right]^{*}$ $=\lim \left\{g_{1}(z) /\left[a_{n}\left(1-r^{2}\right)\right]\right\}$ as $r \uparrow 1$, with $g(z)=f(r z)$. An added remark by the authors is that, if $f(z)$ is self-inversive, no zero of $f^{\prime}(z)$ can lie on the unit circle except at the multiple zeros of $f(z)$. (Received November 10,1950.)

\section{4t. Jean M. Boyer: $A$ note on Fourier constants.}

Let $b_{n}=(2 / M) \sum_{k=1}^{M-1} f(k / M) \sin (n k \pi / M)$ and let $a_{n}=2 \int_{0}^{1} f(x) \sin (n \pi x) d x$. The author shows that if $f(x)$ is integrable and of bounded variation, $\lim _{M \rightarrow \infty} b_{n}(M)=a_{n}$ is uniform in $n$ for $1 \leqq n \leqq M$. (Received November 1, 1950.)

\section{Sarvadaman Chowla: Periodic simple continued fractions.}

Let $N(R)$ denote the number of terms in the period of the simple continued fraction for $R^{1 / 2}$. It is proved that $N(p)$, where $p$ denotes primes, can be arbitrarily large. (Received November 15, 1950.)

106t. A. H. Copeland and Frank Harary: Some embedding properties of rings.

Our object is to find the minimal ring in which the complex numbers and an arbitrary Boolean algebra $B$ can be embedded as a subring and the set of all idempotents respectively. In order to do this, we consider the ring $F$ of all functions which map the points (as defined by the representation theorem) of the Boolean algebra into the complex numbers. This is a ring whose elements are random variables in the sense of Kolmogoroff. $F$ is a ring containing both of these systems but is not minimal. In fact, the idempotents of $F$ constitute the complete extension of $B$. There is a subring of $F$ whose idempotents are the Borel extension of $B$ and another subring whose idempotents are exactly $B$. The latter subring is the desired minimal ring and consists of discrete functions, that is, those which take on only a finite number of values. Both Hilbert spaces and Banach spaces are subsets of the function ring $F$. (Received November 13, 1950.)

107t. D. O. Ellis: Notes on abstract distance geometry. I. The algebraic description of ground spaces.

The terminology of this paper and its abstract will appear very shortly in Publicationes Mathematicae (David Ellis, Geometry in abstract distance spaces, Publicationes Mathematicae Debrecen vol. 2 (1951)). The purpose of the note is to derive from 
certain familiar algebraic properties of the metroid of a ground space the corresponding distance-theoretic properties of the ground space. The methods are very elementary and, for the most part, consist merely of translation between algebraic and distance-theoretic terminology. An interesting example of the results is: If the metroid of a ground space is a nilpotent Abelian group (that is, every element is of period 2) then the ground space is normal; every point forms a complete metric base; and the ground space has the property of triangular fixity. Another result (trivial to show directly) which arises in an unexpected fashion from our results is: The set of nonnegative real numbers forms a groupoid under $|x-y|$ which is neither a quasigroup (hence not a loop) nor a semigroup. (Received October 16, 1950.)

\section{8t. Charles Fox: Iterated transforms.}

This paper obtains generalizations of the iterated transforms $f(x)=\int_{0}^{\infty} e^{-x t} g(t) d t$; $g(x)=\int_{0}^{\infty} e^{-x t} h(t) d t=f(x)=\int_{0}^{\infty}(h(t) /(x+t)) d t$, discussed by Widder in his work on Laplace transforms. The Laplace transform is first generalized and it is then proved that the factors $e^{-x t}$ in the above formulae can be replaced by general Laplace transforms of Fourier kernels. Integral operators are then defined by means of Mellin transforms. In most cases these operators, $T_{1}$ and $T_{2}$, are expressible in the form $T_{1} p\{x\}=\int_{0}^{\infty} p(x u) m(u) d u$ and $T_{2} q\{x\}=\int_{0}^{\infty} q(x u) n(u) d u$, where $m(x)$ and $n(x)$ are a pair of Fourier kernels. Iterated transforms of a very general character are then obtained as follows: $g(x)=\int_{0}^{\infty} T_{1} p\{x t\} h(t) d t ; f(x)=\int_{0}^{\infty} T_{2} q\{x t\} g(t) d t ; f(x)$ $=\int_{0}^{\infty}(p, q)\{t\} h(x t) d t$, where $(p, q)\{t\}=\int_{0}^{\infty} p(u t) q(t) d t$. Further it is shown that when $p(x)=q(x)$ and $m(x)=n(x)$, then there exist Parseval theorems of the type $\int_{0}^{\infty} f(u t) h(t) d t=\int_{0}^{\infty} g(u t) g(t) d t$. The conditions required for the validity of these theorems are mainly that $h(x), p(x)$, and $q(x)$ should belong to $L^{2}(0, \infty)$ and that $M(s)$ and $N(s)$, the respective Mellin transforms of $m(x)$ and $n(x)$, should be bounded on the line $s=1 / 2+i \tau(-\infty<\tau<+\infty)$ and that $M(s) N(1-s)=1$. The investigations are based largely upon the properties of Mellin transforms. (Received November 14, 1950.)

\section{9t. B. R. Gelbaum: A nonabsolute basis for Hilbert space.}

By the use of a construction of M. S. Al'tman (Doklady Akademii Nauk SSSR (N.S.) vol. 69 (1949) pp. 483-485 (Russian)) and an earlier result of the author (Expansions in Banach spaces, Duke Math. J. vol. 17 (1950) pp. 187-196) the existence of a nonabsolute basis in Hilbert space is demonstrated. (Received October 13, 1950.)

\section{0t. A. W. Goodman: Typically-real functions with assigned zeros.}

A function $f(z)=z^{q}+\sum_{n=q+1}^{\infty} b_{n} z^{n}$ is said to be typically-real of order $p$ if it is regular in $|z|<1$, if all the coefficients $b_{n}$ are real, and if there is a $\rho$ such that for $\rho<r<1, \Im f(z)$ changes sign $2 p$ times on the circle $z=r e^{i \theta}$. Let $f(z)$ have zeros $\beta_{1}$, $\beta_{2}, \cdots, \beta_{s}, 0<\left|\beta_{j}\right|<1$, and let $t \geqq 0$ be defined by $q+s+t=p \geqq 1$. An upper bound is obtained for $\left|b_{n}\right|, n=q+1, q+2, \cdots$ in terms of $\left|\beta_{j}\right|$. If $t=0$ or if $t$ is odd this bound is sharp for every $q, s, n$, and $\left|\beta_{j}\right|, j=1,2, \cdots, s$. (Received June 21, 1950.)

111t. Israel Halperin and George Lorentz: Multiply subadditive functions.

Let $S$ be a set with operation $e_{1}+e_{2}, S$ defined for some pairs $e_{1}, e_{2} \in S$. Interesting special cases arise when $e$ are sets and $e_{1}+e_{2}$ is the union of disjoint $e_{1}, e_{2}$, or when $S$ 
is a complemented modular lattice with transitive perspectivity and $e_{1}+e_{2}$ the union of independent elements. The authors discuss functions $W(e)$ on $S$ admitting representations (a) $W(e)=\sup |\phi(e)|$, or (b) $W(e)=\sup \phi(e)$, where sup is taken for a certain family $\{\phi\}$ of functions additive on $S$ (nonnegative in case (b)). The necessary and sufficient condition for (a) is the multiple subadditivity of $W$ (in addition, $W$ must be increasing in case (b)). $W$ is multiply subadditive on a Boolean ring $S$ if $p W(e)$ $\leqq \sum_{r=1}^{n} W\left(e_{r}\right)$ whenever the $e_{r}$ cover $e$ exactly $p$ times, that is, there are disjoint decompositions $e_{r}=\bigcup_{k=1}^{p} e_{r k}, r=1, \cdots, n$, such that $e=\bigcup_{r=1}^{n} e_{r k}, k=1, \cdots, p$, again as disjoint decompositions. (This definition is properly changed for an arbitrary $S$.) For the proof, $S$ is imbedded into the Abelian group $G$ of all formal sums $\sum \pm e_{r}, e_{r} \in S$ (with some identifications); the Hahn-Banach theorem in $G$ is used. (Received October 18, 1950.)

112. P. C. Hammer and Andrew Sobczyk: Critical points of a convex body.

In real $n$-dimensional linear space $L$, let any positive-homogeneous, subadditive functional $p(x)$, positive for $x \neq \theta$, be called a sub-norm. Any bounded convex region $C$ in $L$ with $\theta$ interior to $C$ is the set of $x$ for which a sub-norm $p(x)$ is less than or equal to 1 ; and conversely. Let $y=(y-x)+x, \beta(x-y)+x$, with $\beta \leqq 1$, be boundary points of $C$, and let $r(x)=\max [1 /(\beta+1)]$ for boundary points $y$. Then if $r_{0}$ is the minimum value of $r(x)$, another non-negative function associated with $C$ is $\left(r(x)-r_{0}\right) /\left(1-r_{0}\right)$ $=u(x)$. A point where $r(x)$ is minimum is called a critical point of $C$. For a plane trapezoid, $u(x)$ is not a sub-norm. For a plane triangle, $u(x)$ is the characteristic subnorm with origin at the critical point, and likewise for an $n$-dimensional simplex; for the latter the critical point also is unique. On the other hand, the critical set for a triangular prism is an interval; in general for a region $C$ which is the product of a symmetric $n_{1}$-dimensional region $C_{1}$, and an $n_{2}$-dimensional region $C_{2}$ with unique critical point and ratio $r_{0}>1 / 2, n_{1}+n_{2}=n$, the critical ratio is $r_{0}$ and the critical set is the set of $\left(x_{1}, \theta_{2}\right)$ where $\left\|x_{1}\right\| \leqq\left(2 r_{0}-1\right)$. Conjugate regions and relations with convex linear topological spaces are discussed. (Received November 8, 1950.)

\section{3t. M. H. Heins: Riemann surfaces of infinite genus. I.}

Riemann surfaces of infinite genus with one ideal boundary element which is null in the sense of harmonic measure are considered. By an end of such a surface is meant a subregion whose complement is compact. For the purposes of the present investigation it suffices to consider ends with simple analytic boundary and all further reference to ends is taken in this restrictive sense. Use is made of $R$. Nevanlinna's recent investigations concerning Riemann surfaces with null boundary. Results: (1) There exist admitted surfaces with the property that for each end every nonconstant function meromorphic on the end takes all values infinitely of ten with the exception of at most two (extension of a result of Myrberg). (2) There exist admitted surfaces tolerating nontrivial analytic functions omitting a countable infinity of values. (3) There exist ends tolerating nontrivial bounded harmonic functions not possessing a limit at the ideal boundary. (4) A function analytic and bounded on an end possesses a limit at the ideal boundary, and if the function is not identically constant, it is $(1, m)$ on some subend $(n \geqq 2)$. (Received November 6, 1950.)

114t. M. H. Heins: Riemann surfaces of infinite genus. II.

This is a continuation of Part I. (5) Let the family of non-negative harmonic functions on an end which vanish continuously on the relative boundary be intro- 
duced. If it is generated by a finite subset of its members (the family being considered as an additive semimodule with operator), the least number of generators is termed the dimension of the end. Otherwise the dimension is taken as $\infty$. Dimension is independent of ends of an admitted surface and is a conformal invariant of the surface. (6) For each end of an admitted surface the bounded harmonic functions whose domain is the given end all possess limits at the ideal boundary if and only if the dimension is one. (7) If $n$ of (4) is taken as least possible, then the dimension does not exceed $n$. (8) There exist two-sheeted transcendental hyperelliptic Riemann surfaces tolerating harmonic functions which are nontrivial, singularity-free, and $O(\log r)$. (Received November 6, 1950.)

115. Edwin Hewitt and H. S. Zuckerman: Equivalence of two notions of integral.

Let $X$ be a locally compact Hausdorff space, and let $\mathfrak{C}_{+}$be the set of all continuous, real-valued, non-negative functions on $X$ each of which vanishes outside of some compact set. Let $M$ be a real-valued, non-negative functional defined on $\mathfrak{S}_{+}$such that $M(f+g)=M(f)+M(g)$ for $f, g \in \mathbb{C}_{+}$and $M(\alpha f)=\alpha M(f)$ for $\alpha$ real and greater than or equal to 0 and $f \in \mathfrak{C}_{+}$. For all compact subsets $A$ of $X$, define $\mu(A)$ as $\sup _{0} \leqq_{f} \oint_{A} M(f)$. For all open subsets $G$ of $X$, define $\mu^{*}(G)$ as sup $\mu(A)$, taken over all compact $A \subset G$. For all subsets $P$ of $X$, define $\mu_{*}(P)$ as inf $\mu_{*}(G)$, taken over all open $G \supset P$. It is proved that $\mu^{*}$ is an outer measure on $X$ in the sense of Caratheodory with respect to which every compact set and every open set of finite $\mu_{*}$-measure is measurable. Following Daniell, Bourbaki, Stone, and McShane, one defines a class of summable functions on $X$ with respect to $M$ by using the classes of upper and lower semi-continuous functions on $X$, in the usual fashion. It is proved that a function is summable if and only if it is measurable $\left(\mu^{*}\right)$ and has a finite integral $\left(\mu^{*}\right)$. In this case, the Daniell sum is equal to the $\mu^{*}$-integral. (Received November 17,1950 .)

116t. R. C. James: A nonreflexive separable Banach space isometric with its second conjugate space.

For a sequence $x=\left(x_{1}, x_{2}, \cdots\right)$, let $\|x\|=1$.u.b. $\left[\sum_{i=1}^{n}\left(x_{p_{i}}-x_{p_{i+1}}\right)^{2}+\left(x_{p_{n+1}}\right.\right.$ $\left.\left.-x_{p_{1}}\right)^{2}\right]^{1 / 2}$, where the 1.u.b. is over all positive integers $n$ and finite increasing sequences of positive integers $p_{1}, p_{2}, \cdots, p_{n+1}$. Let $B$ be the Banach space of all $x$ for which $\lim _{n \rightarrow \infty} x_{n}=0$ and $\|x\|$ is finite. This space $B$ is isomorphic with a nonreflexive Banach space which is known to be isomorphic with its second conjugate space and which is isometric under the natural mapping with a maximal closed linear subspace of its second conjugate space. $B^{* *}$ is the space of all $F=\left(F_{1}, F_{2}, \cdots\right)$ for which $\|F\|$ $=\lim _{n \rightarrow \infty}\left\|\left(F_{1}, \cdots, F_{n}, 0,0,0, \cdots\right)\right\|$ is finite. The mapping $x=\left(x_{1}, x_{2}, \cdots\right)$ $\leftrightarrow F_{x}=\left(x_{2}-x_{1}, x_{3}-x_{1}, \cdots, x_{n}-x_{1}, \cdots\right)$ is an isometry of $B$ with $B^{* *}$. (Received November 13, 1950.)

\section{S. L. Jamison: Perturbation of normal operators. Preliminary} report.

A normal operator $N$ on a Hilbert space is a closed, densely defined linear transformation of the space into itself which commutes with its adjoint, that is, $N^{*} N$ $=N N^{*}$. If a bounded operator which depends on a real parameter $\epsilon$ is expressible as a convergent power series in $\epsilon: T(\epsilon)=T_{0}+\epsilon T_{1}+\epsilon^{2} T_{2}+\cdots$ where $T_{0}, T_{1}, T_{2}, \cdots$ are bounded operators, then $T(\epsilon)$ is said to depend regularly on $\epsilon$. An isolated portion of the spectrum of a regular normal operator $N(\epsilon)$ not only moves continuously 
with $\epsilon$ but a measure of how fast it moves is obtained. In particular, if the unperturbed operator $N(0)=N_{0}$ possesses an isolated eigenvalue $\lambda_{0}$ of finite multiplicity $m$, then there are $m$ numerical functions $\lambda^{(i)}(\epsilon)$ and $m$ elements $\phi^{(i)}(\epsilon)$ (depending regularly on $\epsilon$ ) such that the $\lambda^{(i)}(\epsilon)$ are eigenvalues of the perturbed operator $N(\epsilon)$, $\lambda^{(i)}(0)=\lambda_{0}$, and the $\phi^{(i)}(\epsilon)$ are corresponding mutually orthogonal normed eigenelements. These results generalize those of Franz Hellich (Math. Ann. vol. 113 (1936)) and Bela v. Sz. Nagy (Comment. Math. Helv. vol. 19 (1947)) who proved them for self-adjoint operators. A first method of proof is a reduction to the selfadjoint case, and a second method is a generalization of Nagy's techniques. (Received November 9, 1950.)

\section{R. V. Kadison: Isometries in operator algebras.}

An isometry (norm preserving linear isomorphism) of $C^{\prime}(X)$, the set of all continuous complex-valued functions on the compact-Hausdorff space $X$, is a ring isomorphism of $C^{\prime}(X)$ followed by multiplication by a unitary function. The noncommutative extension of this result to arbitrary $C^{*}$-algebras (uniformly closed, selfadjoint algebras of operators on a Hilbert space containing an identity) is proved. Specifically, if $\mathfrak{A}_{1}$ and $\mathfrak{A}_{2}$ are $C^{*}$-algebras and $\rho$ is an isometry between them, then $\rho$ is the composition of a linear isomorphism which preserves the adjoint operation and is multiplicative on powers ( $C^{*}$ or quantum isomorphism) with left multiplication by a unitary operator of $\mathfrak{A}_{2}$. The converse, that any such map is an isometry, is also proved. As a preliminary to these results all extreme points on the unit sphere of a $C^{*}$-algebra $\mathfrak{A}$ are determined. They are those partially isometric operators $U$ in $\mathfrak{A}$ such that $(I-F) \mathfrak{A}(I-E)=(0)$ where $U^{*} U=E$ and $U U^{*}=F$. In the case of weakly closed $C^{*}$-algebras (rings of operators), the form of $C^{*}$-isomorphisms is studied. Applications are made to factors. It is proved for example that factors that are isometric (equivalent) as Banach spaces are ${ }^{*}$ isomorphic as algebras. (Received November 6, 1950.)

\section{Joseph Kampé de Fériet: Autocorrelation function of a trun-} cated function.

In a previous paper (to be published in the Proceedings of the International Congress of Mathematicians) the author has given a new way to obtain the energy spectrum (defined by Norbert Wiener, Acta Math. vol. 55 (1930) pp. 117-258) of a function $u(t)$ which belongs to $L^{2}$ for every finite interval and has a continuous autocorrelation function; his method is based on the definition of an autocorrelation function $\rho_{T}(h)$ for the truncated function $u_{T}(t)$ (equal to $u(t)$ in $|t| \leqq T$ and to zero outside). The present paper establishes that $\rho T(h)$, which is zero for $|h| \geqq 2 T$, is represented in $|h| \leqq 2 T$ by the absolutely and uniformly convergent Fourier series: $\rho_{T}(h)$ $=\sum_{-\infty}^{+\infty}\left|a_{n}\right|^{2} \exp (n \pi i h: 2 T)$, where $a_{2 n}$ and $a_{2 n+1}$ are respectively the $n$th Fourier constant of $u_{T}$ and of $u_{T} \exp (-\pi i t: 2 T)$. From this it follows that not every convergent trigonometrical series with positive coefficients can represent an autocorrelation function of a truncated function: one can choose arbitrarily only the even (odd) coefficients (provided that their sum converges). (Received November 13,1950.)

120. M. S. Klamkin: Sums and asymptotic sums of series related to the harmonic series.

Various elementary identities are derived and are applied to determine sums and asymptotic sums of series related to the harmonic series. Among the series summed 
are the following whose $p$ th terms are: $S_{1, p} / p ; S_{1, p} / p^{2} ; S_{1, p}^{2} / p ; S_{1, p}^{3} / p ; S_{1, p}^{2} ; S_{2, p} / p ; S_{2, p} / p^{2}$; $S_{3, p} / p ; S_{1, p} / p^{3} S_{1, p} S_{2, p} / p ; S_{n, p}$ where $S_{m, p}=\sum_{r=1}^{p}\left(1 / r^{m}\right)$. (Received November 13,1950 .)

\section{R. J. Levit: A generalization of the Jacobi polynomials orthog-} onal on a finite set of points.

The Legendre, Hermite, and Laguerre polynomials are limiting cases of certain finite systems of polynomials which satisfy orthogonality conditions expressed in terms of finite sums (references in G. Szegö, Orthogonal polynomials, Amer. Math. Soc. Colloquium Publications, vol. 23, \$2.8, 2.9). In the present paper a system of polynomials $p_{n}^{(h, \alpha, \beta)}(x)$ of degrees $n=0,1, \cdots, N-1$ is defined which is a similar sense constitutes a finite generalization of the Jacobi polynomials. More precisely, the polynomials $p_{n}^{(h, \alpha, \beta)}(x)$ satisfy orthogonality conditions of the form $\sum_{i=0}^{N^{-1}} w^{(h, \alpha, \beta)}\left(x_{i}\right)$ - $p_{m}^{(h, \alpha, \beta)}\left(x_{i}\right) p_{n}^{(h, \alpha, \beta)}\left(x_{i}\right)=0$ for $m \neq n$, where $w^{(h, \alpha, \beta)}(x)$ is a. certain positive function on the finite set $x_{0}<x_{1}<\cdots<x_{N-1}$; and, whenever $\alpha>-1, \beta>-1, p_{n}^{(h, \alpha, \beta)}(x)$ approaches the Jacobi polynomial $P_{n}^{(\alpha, \beta)}(x)$ while $w^{(h, \alpha, \beta)}(x)$ approaches the Jacobi weight function $(1-x)^{\alpha}(1+x)^{\beta}$ as $h \rightarrow 0$. The usual properties of orthogonal systems, such as a recurrence relation, formula of Rodrigues' type, and so forth, are developed. For certain values of $h, \alpha, \beta$ the polynomials $p_{n}^{(h, \alpha, \beta)}(x)$ continue to form an orthogonal system even though the inequalities $\alpha>-1, \beta>-1$ do not hold. In some of these cases they exhibit the peculiarity of being orthogonal on two distinct sets of points with respect to $w^{(h, \alpha, \beta)}(x)$. (Received November 13, 1950.)

122t. A. J. Lohwater: Boundary values of functions analytic in the unit circle.

The author shows that, if $f(z)$ is analytic with no zeros in $|z|<1$, and if $f(z)$ possesses radial limit values of modulus 1 for almost all $e^{i \theta}$ of $|z|=1$, then, in order that $f(z)$ not be identically constant, there must exist a path interior to $|z|<1$ and terminating at a point of $|z|=1$ along which either $f(z) \rightarrow 0$ or $f(z) \rightarrow \infty$. If there is no path along which $f(z) \rightarrow \infty$, then $|f(z)|<1$ in $|z|<1$. If, in addition to the above hypotheses, $f(z)$ is of bounded characteristic in $|z|<1$, the path mentioned above becomes radial. A uniqueness theorem for harmonic functions of bounded mean modulus follows as a corollary. (Received October 26, 1950.)

\section{3t. A. N. Milgram and P. C. Rosenbloom: Heat conduction on} Riemannian manifolds. II. Properties of solutions.

In their previous abstract (Bull. Amer. Math. Soc. Abstract 57-1-4) the authors discussed existence and certain properties of the solutions of the equations (1) $\Delta \alpha=\partial \alpha / \partial t$ where $\alpha$ is an exterior differential form defined on a closed orientable Riemannian manifold of class $C^{2}$. Let $T_{t} \alpha$ denote the solution at time $t$ having initial value $\alpha$. A spectral analysis of the complete semigroup of transformations $T_{t}$ is made by elementary means. As a corollary, the decomposition theorem of deRham and the theories of the equations $\Delta \alpha=\lambda \alpha$ and $\Delta \alpha=\beta$, where $\beta$ is a given form, are obtained. $T_{t} \alpha$ can be represented as the scalar product of $\alpha$ with the double form $J_{p}(x, y, t)$ which is the so-called fundamental solution of the equation (1). The integral equation for $T_{t} \alpha$ leads immediately to the series representation of $J_{p}$ and makes it possible to deduce readily that the solutions of (1) have the same degree of smoothness as the manifold. This integral representation of $T_{t}$ shows that this operator is completely continuous. $J_{p}$ behaves like the euclidean fundamental solution as $t \rightarrow 0$. (Received November 13, 1950.) 


\section{4t. K. S. Miller: Self-adjoint factorizations of differential operators.}

In this short note, the following theorem is proved: Let $L$ be an ordinary linear differential operator, $L u=p_{0}(x) u^{(n)}+p_{1}(x) u^{(n-1)}+\cdots+p_{n}(x) u$ of even order $n=2 r$. $p_{i}(x) \in C^{n-i}$ and $p_{0}(x)>0$ in some closed finite interval $[a, b]$. Then there exists a subinterval of $[a, b]$ in which $L$ has a representation $L=f(x) P_{1} P_{2} \cdots P_{r}$ where each $P_{\alpha}, \alpha=1,2, \cdots, r$, is a linear differential operator of the second order and formally self-adjoint. (Received October 30, 1950.)

125. Josephine M. Mitchell: On the spherical summability of multiple orthogonal series.

Let $\left\{\boldsymbol{\phi}_{m}\right\}\left(m=m_{1} \cdots m_{q} ; m_{k}=0,1,2, \cdots ; k=1, \cdots, q\right)$ be a complete orthonormal system of real Lebesgue square integrable functions defined on a measurable set $E \subset q$-dimensional Euclidean space. Let $\sum a_{m} \phi_{m}$ be a multiple orthogonal series, where $\left\{a_{m}\right\}$ is a set of constants satisfying $\sum a_{m}^{2}<\infty$. We consider the (spherical) convergence and $(C, \alpha)$ summability of the orthogonal series, that is, respectively, the existence of $\lim _{N \rightarrow \infty} S_{N}$, where $S_{N}=\sum_{\nu=0}^{N} 0_{\nu}, 0_{\nu}=\sum a_{m} \phi_{m}$ if $\nu=m_{1}^{2}+\cdots+m_{q}^{2}$ or 0 if $\nu \neq m_{1}^{2}+\cdots+m_{q}^{2}$, and of $\lim _{N \rightarrow \infty} \sigma_{N}^{\alpha}$, where $\sigma_{N}^{\alpha}=\left(p_{N}^{\alpha}\right)^{-1} \sum_{\nu-0}^{N} p_{N-\nu}^{\alpha} 0_{\nu}(\alpha>0)$, $p_{N}^{\alpha}=(\alpha+1) \cdots(\alpha+N) / N$ !. By means of methods similar to those used in the case $q=1$, it is possible to extend all the well known sufficient conditions for simple orthogonal series to the convergence and $(C, \alpha)$ summability of the multiple series $\sum a_{m} \phi_{m}$. In addition we prove that the $(C, \alpha)$ summability methods are all equivalent for $\alpha>0$ and equivalent to the Riesz mean summability of order $\alpha$ and type $\nu$. These results represent a considerable improvement over known results of the same type on spherical convergence and summability of multiple Fourier series. (Received November 17, 1950.)

126. C. N. Moore: On convergence factors for summable double series whose Cesàro means do not remain bounded.

It is usual in dealing with convergence factors in convergent or summable double series to make the assumption that the partial sums or the corresponding means of partial sums remain bounded. This is the case of greatest interest and the one which yields the simplest results. However, for the sake of completeness, it seems desirable to investigate the modifications in the theory which result from dropping this assumption. The present paper deals with the situation in question for the case of Cesaro summable double series. (Received November 14, 1950.)

\section{7t. G. D. Mostow: On the $L^{2}$-system of a Lie group.}

Theorem. Suppose $G$ is a connected Lie group. Then there is a compact subgroup $K \subset G$ and an analytic submanifold $E$ of $G$ such that (1) the mapping $(k, e) \rightarrow k \cdot e$ of the analytic manifold $K \times E$ onto $G$ is an analytic isomorphism (that is, analytic mapping with nonvanishing Jacobian); (2) coordinates can be introduced on $E$ in such a way that Haar measure on $G$ becomes the product measure of Haar measure on $K$ and ordinary euclidean measure on $E$. From (1) we conclude that a Lie group can be embedded analytically in euclidean space. From (2) we conclude that the $L^{2}$-system of $G$ (with respect to Haar measure) admits a base of functions which are analytic on G. (Received November 10,1950.)

\section{8t. Zeev Nehari: Some extremal maps.}

Let $D$ be a multiply-connected domain in the complex z-plane and let $S$ be the 
class of univalent functions $w=f(z)$ in $D$ which have a simple pole of residue 1 at a given point $\zeta$ of $D$. If $a, b$ are two distinct points of $D$, the region of variability $\Delta$ of the expression $\log \{f(a)-f(b)\}(f(z) \in S)$ is found and a geometric characterization of the conformal maps associated with the boundary points of $\Delta$ is given. In particular, it is shown that the function $f_{0}(z)$ solving the problem $|f(a)-f(b)|=\max$ maps $D$ onto a slit domain whose slits are confocal elliptic arcs with the foci $f_{0}(a), f_{0}(b)$. The problem $|f(a)-f(b)|=$ min leads to a corresponding hyperbolic slit domain. Results of the same type are also obtained for the domain of variability of $\log \{f(a) f(b)\}$. (Received November 13, 1950.)

\section{G. O. Peters: Note on Sterling's numbers. Preliminary report.}

The author uses the definition $x^{(n)}=x(x-1) \cdots(x-[n-1])$, then $x^{(n)}$ can be expressed as a polynomial in powers of $x$, and $x^{n}$ as a polynomial in factorials of $x$, using Sterling's numbers of the first and second kind respectively. The author then uses the definition $x^{(-n)}=1 /(x+1)(x+2) \cdots(x+n)$ and expresses $x^{(-n)}$ as a power series, and expresses $x^{-n}$ as a factorial series, and calls the coefficients in these series Sterling's numbers of the first and second kind of negative degree. The Sterling's numbers of the first and second kind may thus be defined by $x^{(n)}=\sum_{\nu=n}^{0} S_{n}^{\nu} x^{\nu}$ and $x^{n}=\sum_{\nu-n}^{0} \zeta_{n}^{\nu} x^{(\nu)}$. And the Sterling's numbers of the first and second kind of negative degree may be defined by $x^{(-n)}=\sum_{\nu_{-n}}^{\infty} S_{-n}^{-\nu} x^{-\nu}$ and $x^{-n}=\sum_{\nu_{-n}}^{\infty} \zeta_{-n}^{-\nu} x^{(-n)}$. The author finds a simple relation between the well known Sterling's numbers of positive degree and the Sterling's numbers of negative degree, given by $S_{-k}^{-l}=(-1)^{l+k} \xi_{\imath}^{k}$ and $\zeta_{-k}^{-l}=(-1)^{l+k} S_{l}^{k}$. (Received November 14, 1950.)

130t. M. O. Reade: On Gustin's bilinear integral operator. Preliminary report.

Gustin's bilinear integral identity for harmonic functions [Amer. J. Math. vol. 70 (1948) pp. 212-220] is used to obtain information concerning subharmonic functions. A typical result is the following one. Let $f(x, y)$ be subharmonic for $x^{2}$ $+y^{2}<1$, and let $\mu(e)$ be the non-negative mass distribution associated with $f(x, y)$. Then for each triple of positive constants $\lambda_{k}, k=1,2,3, \lambda_{1} \lambda_{2}=\lambda_{3}^{2}$, $\lim _{r \rightarrow 0}\left\{\left(2 / \pi r^{2}\right) \int_{0}^{2 \pi}\left[f\left(x_{0}+\lambda_{1} r \cos \phi, y_{0}+\lambda_{1} r \sin \phi\right) f\left(x_{0}+\lambda_{2} r \cos \phi, y_{0}+\lambda_{2} r \sin \phi\right)\right.\right.$ $\left.-f^{2}\left(x_{0}+\lambda_{3} \cos \phi, y_{0}+\lambda_{3} r \sin \phi\right)\right] d \phi=\left(\lambda_{1}-\lambda_{2}\right)^{2} f\left(x_{0}, y_{0}\right) D_{8} \mu\left(x_{0}, y_{0}\right)$ holds almost everywhere in $x^{2}+y^{2}<1$. (Received November 13, 1950.)

\section{P. K. Rees: Rotation of isometric circles in transforms of} Fuchsian groups.

This paper gives several theorems concerning the angles $\operatorname{gog}_{t}=\theta_{t}$ and $\operatorname{gog}_{s}=\theta^{s}$ where $g, g_{t}$, and $g_{s}$ are the centers of the isometric circles of the Fuchsian transformations $G(z)=(\alpha z+\bar{\nu}) /(\nu z+\bar{\alpha}), \alpha \bar{\alpha}-\nu \bar{\nu}=1 ; T(z)=(a z+\bar{c}) /(c z+\bar{a}), a \bar{a}-c \bar{c}=1 ;$ and $S(z)$ $=G T G^{-1}=(A z+\bar{C}) /(C z+\bar{A}), A \bar{A}-C \bar{C}=1$. The transformation $G$ is considered as variable, and $T$ is any fixed transformation of a group. The coefficients $\alpha$ and $\nu$ are restricted to real values so as to lead to a simple geometric situation, but $a, c, A$, and $C$ are complex. The following is typical of the theorems: If the imaginary part of $a c$ is different from zero, then the necessary and sufficient condition that $\sin \theta_{s}=0$ is that the center $g=-\bar{\alpha} / \nu=-\alpha / \nu$ of the isometric circle of $G$ be at an intersection of the real axis and the fixed circle $Q_{8}$ of $T$ with center at $[(a+\bar{a})(a-\bar{a})+(c+\bar{c})$ $(c-\bar{c})] / 2(a c-\overline{a c})$ and radius $\left[(a+\bar{a})(a-\bar{a})+(c+\bar{c})(c-\bar{c})-4(a c-\overline{a c})^{2}\right]^{1 / 2} / 2(a c-\overline{a c})$. (Received November 13, 1950.) 
132. J. J. Sopka: Reynolds operators on the algebra of all continuous functions on a compact Hausdorff space.

A Reynolds operator $T$ on an algebra $A$ with a unit is an endomorphism of $A$ which takes the unit into itself and which has the property: $T(f T(g))=T(f) T(g)$ for all $f$ and $g$ in $A$. If an order relation $\geqq$ is defined in $A, T$ is called order-preserving if $f \geqq 0$ implies $T(f) \geqq 0$. In the case where $A=C(K)$, the family of all continuous functions on a compact Hausdorff space $K$, then to each order-preserving Reynolds operator $T$ on $C(K)$ there corresponds a partition $K$ of $K$ into closed subsets $K^{\alpha}$ of $K$, and a family of Radon measures $\left\{\mu_{\alpha}\right\}$, one $\mu_{\alpha}$ defined in each $K^{\alpha}$, such that for each $f$ in $C(K)$ the value of $T(f)$ is constant on each $K^{\alpha}$ and equal to the integral of $f$ over $K^{\alpha}$ with respect to the measure function $\mu_{\alpha}$. A necessary and sufficient condition is given for a system $\left[K, K,\left\{\mu_{\alpha}\right\}\right]$ to admit a Reynolds operator. (Received November 10, 1950.)

\section{3t. Otto SzÁsz: Identities and inequalities concerning orthogonal} polynomials and Bessel functions.

Several papers have been written recently generalizing a nonlinear inequality concerning Legendre polynomials, due to $P$. Turán. In these papers analogous inequalities were derived for ultraspherical polynomials, Laguerre and Hermite polynomials, and for Bessel functions. In the present paper some new proofs and further improvements of these results are given. The methods are based again partly on recursion formulas and partly on linear differential equations, satisfied by the functions under consideration. (Received November 3,1950.)

\section{T. T. Tanimoto: Manifolds in Hilbert space.}

Let $X$ be an abstract real Hilbert space with inner product $x_{1} \times x_{2}$, and $u \in X$ a vector function of vectors $x_{1}, x_{2}, \cdots, x_{r} \in X$ linear in all $x^{\prime}$ s whose domains are $X$. Call $u$ an $r$-hyperlinear transformation $\alpha^{[r]}$. Continuity, boundedness, and their equivalence are developed in the usual way. Let $\sigma_{(q)}$ be a transformation of $X$ into $X$, not necessarily linear, by a function of a point $q$. (Proper distinctions are made between vectors and points.) The derivatives of $\sigma_{(q)}$ with respect to $q$ are defined conventionally and are linear or hyperlinear operators. A manifold is defined as the range of $\sigma_{(q)}$. The tangent linear manifold $M_{q}$ at $q$ is defined to be the range of $\alpha_{(q)}=d \sigma_{(q)} / d q$. Nonsingular points with usual properties are defined. If $E$, a function of $q$, projects any vector on $M_{q}$ and if $\beta_{(q)}^{[r]}$ is an $r$-hyperlinear operator, a function of $q$, the intrinsic differential, if $d \beta_{(q)}^{[r]}$ exists, is defined by $d^{*} \beta_{(q)}^{[r]}=E d \beta_{(q)}^{[r]}$. $d E$ is not proved to exist but we define $d^{*} E=E\left(\alpha^{-1}\right)_{-1}(d \alpha)_{-1}(1-E)$ where $\left(\alpha^{-1}\right)_{-1},(d \alpha)_{-1}$ are the adjoints of $\alpha^{-1}$ and $d \alpha$ and where $\alpha=d \sigma / \alpha q$. If $d_{1} q$ and $d_{2} q$ are distinct vectors at $q$ in $M_{q}$ we define the Riemann tensor by $R{ }^{[3]} d_{1} q d_{2} q u=d_{1}^{*} d_{2}^{*} u-d_{2}^{*} d_{1}^{*} u$ for any vector $u$ at $q$ in $M_{q}$. The curvature is defined by $K=R^{[3]} b_{1} b_{2} b_{1} \times b_{2} /\left(b_{1}^{2} b_{2}^{2}-\left(b_{1} \times b_{2}\right)^{2}\right)$, where $b_{1}$ and $b_{2}$ are distinct unitary vectors at $q$ in $M_{q}$. (Received November 13,1950 .)

135. H. E. Taylor: On the type and form of the mapping function of certain Riemann surfaces. Preliminary report.

Three classes of Riemann surfaces are defined, all open and with four firstorder branch points in each sheet $S_{n}$ on the real axis over $w=a_{n}, b_{n}, a_{n-1}, b_{n-1}$. For class I, $0<a_{1}<b_{1}, a_{2 k \pm 1}>a_{2 k}, b_{2 k-1}>a_{2 k-1}, a_{2 k}>b_{2 k}, k=1,2,3, \cdots$. For class II, $b_{-1}<a_{-1}<0<a_{1}<b_{1}, a_{2 k \pm 1}>a_{2 k}, b_{2 k-1}>a_{2 k-1}, a_{2 k}>b_{2 k}, a_{-2 k}>a_{-2 k \pm 1}, b_{-2 k}>a_{-2 k}, a_{-2 k-1}$ $>b_{-2 k-1}, k=1,2,3, \cdots$. For class III, $0<a_{1}<b_{1}<b_{2}, b_{2 k}<a_{2 k}<a_{2 k+1}<b_{2 k+1}<b_{2 k+2}$, 
$k=1,2,3, \cdots$. Any surface of class I or III is parabolic and any surface of class II is the image of the $z$-sphere less two points. The meromorphic function $w=f(z)$ corresponding to surfaces of class I is such that $f^{\prime}(z)=e^{\hat{\delta}_{z}} \prod_{k=1}^{\infty}\left(1-z / \alpha_{k}\right)\left(1-z / \beta_{k}\right) /\left(1-z / \gamma_{k}\right)$ with $\delta \leqq 0 ; \alpha_{k},-\beta_{k},-\gamma_{k}$ all $\rightarrow \infty$ as $k \rightarrow \infty ; \beta_{k}<\gamma_{k}<\beta_{k-1}<0<\alpha_{k}<\alpha_{k+1}$; and with the residues of $f^{\prime}(z)$ at the poles all zero. A partial converse of this result is obtained. Analogous results are obtained for surfaces of class II. The methods employed involve approximation of the given surfaces by sequences of elliptic surfaces as developed by G. R. MacLane (Trans. Amer. Math. Soc. vol. 62 (1947) pp. 99-113); the use of the theory of normal families of functions; and the results of Caratheodory (Math. Ann. vol. 72 (1912) pp. 107-144) on the mapping of a sequence of plain domains by a family of functions. (Received November 9, 1950.)

\section{6t. F. G. Tricomi: A class of nonorthogonal polynomials related to} those of Laguerre.

A systematic study is made of the polynomials $l_{n}(x)=(-1)^{n} L_{n}^{(x-n)}(x)$ and of the more general ones in which the upper index of the Laguerre polynomial is $x-y-n$, which appear as coefficients in some important expansions. These polynomials (for which the degree of $l_{n}$ in $x$ is the greatest integer in $n / 2$ ) have a simple generating function and the recurrence relation $(n+1) l_{n+1}-(n+y) l_{n}+x l_{n-1}=0$ holds. Further its zeros are all real and the $k$ th of them $(k=1,2, \ldots$ fixed) is a decreasing function of $n$ which approaches the limit $y+k-1$ as $n \rightarrow \infty$. An asymptotic expansion of $l_{n}$ for $n \rightarrow \infty$ and $x$ and $y$ bounded is found and some further properties established. The generalized polynomial is a particular solution of the parabolic partial differential equation $(\xi+\eta) u \xi+(\eta-\xi) u \xi+n u=0$ where $x=\xi+\eta, y+n-1=\xi-\eta$, which is closely related to the differential equation of the confluent hypergeometric functions. (Received November 9, 1950.)

\section{7t. H. S. Wall: $J$-matrices of interior order $k$. Preliminary report.}

Let $u_{n}=\left(u_{n}^{i j}\right)$ be a $k$ by $k$ matrix of polynomials in $x$ of degree $\leqq n,=n,<n$, according as $i<j, i=j, i>j$, respectively, and $\Phi$ a $k$ by $k$ symmetric matrix of functions in $B V[-\infty,+\infty]$ such that $\int u_{m}^{\prime} d \Phi u_{n}=\delta_{m n} \cdot I, m, n=0,1,2, \cdots$ If $C_{p}=\int x^{p} d \Phi$, $p=0,1,2, \cdots,\left\{u_{n}\right\}_{n=0}^{\infty}$ exists only if the determinants of the segments of the matrix $\left(C_{i+j}\right)$ are different from 0 . There exist sequences $\left\{A_{p}\right\}_{p-1}^{\infty},\left\{B_{p}\right\}_{p=1}^{\infty}$ of $k$ by $k$ matrices of numbers, $A_{p}$ nonsingular with only 0 's above the main diagonal, $B_{p}$ symmetric, such that $u_{n-1} A_{n}+u_{n}\left(B_{n+1}+x I\right)+u_{n+1} A_{n+1}^{\prime}=0, n=0,1,2, \cdots, u_{-1}=0$. The matrix $J$ of the quadratic form $\sum\left(\xi_{p}^{\prime} B_{p} \xi_{p}+\xi_{p}^{\prime} A_{p} \xi_{p+1}+\xi_{p+1}^{\prime} A_{p} \xi_{p}\right), \xi_{p}$ a $k$-dimensional column vector, is called a $J$-matrix of interior order $k$. A substantial part of the theory of containued fractions $(k=1)$ is extended to $J$. A reciprocal $r(\rho)$ of $J+z I$ is determined by its $k$ th segment $r(\rho)_{k}$, which is an arbitrary symmetric $k$ by $k$ matrix $\rho$. The $k$ th segment $f_{m}$ of the reciprocal of $(J+z I)_{m k}$ plays the role of $m$ th approximant. If $J(J+z I)$ $>0$ for $J(z)>0$, there exists for $J(z)>0$ a bounded $r(\rho)=\lim r\left(f_{m}\right)$ as $m \rightarrow \infty$ over suitable values, and $\rho=\rho(z)=\int d \Phi(x) /(z-x)$, the quadratic form $\xi^{\prime} \Phi \xi$ a nondecreasing function of $x$. Application is made to the moment problem of Boas and Polya. (Received October 13, 1950.)

138. H. F. Weinberger and Alexander Weinstein: On the connection between the eigenvalues and eigenfunctions of some Sturm-Liouville problems.

Using methods previously developed by one of the authors (A. Weinstein, Reissner 
anniversary volume, Ann. Arbor, Edwards, 1949, pp. 404-414), explicit expressions for the eigenvalues and eigenfunctions of the Sturm-Liouville problem $u^{\prime \prime}-r(x) u+\lambda u=0$ with periodic boundary conditions are given in terms of the eigenvalues and eigenfunctions of the same equation with the boundary conditions $u^{\prime}=0$. A generalization of this process leads to similar results for the fourth order Sturm-Liouville problem $\left(k(x) u^{\prime \prime}\right)^{\prime \prime}-\lambda \rho(x) u=0$ with any self-adjoint boundary conditions. The eigenvalues and eigenfunctions of this problem with the added condition $\left(^{*}\right): u=0$ at a given interior point are obtained in terms of the Green's function (and hence of the eigenvalues and eigenfunctions) of the same problem without the condition $\left({ }^{*}\right)$. This result has application to the theory of continuous beams. (Received October 25, 1950.)

\section{J. G. Wendel: On isomorphism of group algebras.}

The classes of real- and complex-valued integrable functions $x(g)$ on a locally compact group $G$ form Banach algebras, $L(G)$. It is shown in both the real and complex cases that two such algebras $L(G)$ and $L(\Gamma)$ are isometrically and algebraically isomorphic if and only if the groups are topologically isomorphic. The relation between the algebra isomorphism $T$ and the group isomorphism $\tau$ is given by the formula $(T x)(\tau g)=c \chi(g) x(g), x \in L(G), g \in G, T x \in L(\Gamma), \tau g \in \Gamma$, where $c$ is a constant, $\chi(g)$ is a continuous character on $G$. The proof uses the left translation operators on $L(G)$, and exploits methods due to Kawada (Mathematica Japonicae vol. 1 (1948) pp. 1-5). (Received November 8, 1950.)

\section{A. D. Ziebur: The asymptotic solutions of a certain type of ordi-} nary differential equation of the second order.

This paper is concerned with the solutions of the differential equation (1) $d^{2} u / d z^{2}$ $+\left[\lambda^{2} P(z)^{2} / z+a_{2} / z^{2}+a_{1} / z+\sum_{i=0}^{\infty} q_{i}(z) / \lambda^{i}\right] u=0$, where $\lambda$ is a large complex parameter, $a_{1}$ and $a_{2}$ are arbitrary constants, and $P(z)$ and the $q_{i}(z)$ are analytic in some bounded domain $R_{z}$ which contains the origin. It is supposed that the above series converges for all $\lambda$ greater than some fixed value and that $P(z)$ is nonvanishing in $R_{z}$. An algorithm for the determination of the coefficients of a formal series in inverse powers of $\lambda$, segments of which serve to represent solutions of equation (1) asymptotically, is developed, and the asymptotic forms of certain particular solutions are determined. The Stokes phenomenon is avoided by the admission of Bessel functions into the asymptotic representations. The methods used are essentially those of R. E. Langer (Trans. Amer. Math. Soc. vol. 67 (1949) pp. 461-490). The confluent hypergeometric function $M_{k, m}(z)$ of Whittaker is a solution of a particular equation of the form (1), and by specializing the results obtained for the more general case, asymptotic expansions of $M_{k, m}(z)$ with respect to $k$ are obtained. (Received October 30,1950.)

\section{1t. H. J. Zimmerberg: On normalizable transformations in Hilbert} space.

The existence theorems for normalizable transformations in Hilbert space developed by Zaanen (Acta Math. vol. 83 (1950) pp. 197-248) are extended and applied to systems of definite Fredholm integral equations previously considered by the author (Duke Math. J. vol. 15 (1948) pp. 371-388). (Received November 9, 1950.)

\section{ApPlied mathematics}

142. O. R. Ainsworth: Theory of waves from a point source in one of 
two contiguous semi-infinite isotropic elastic media. Preliminary report.

Two isotropic elastic media, one occupying $z>0$ and the other $z<0$, are considered. First a $P$-wave instantaneous point source with $S$ waves occurring in the reflected and transmitted fields is considered. By symmetry the $S$ waves are all oriented perpendicular to $z=0$. Making the stresses and displacements continuous across $z=0$, evaluate the undetermined functions, and the contour integrals for the displacements are reduced to definite integrals of algebraic quantities by applying a theorem of E. Pinney. The $S$-wave sources, oriented parallel and perpendicular to $z=0$, are also considered separately, and, again, the displacements are given as definite integrals. (Received November 15, 1950.)

\section{Cahit Arf: On a free boundary problem in elasticity.}

The author considers the free boundary problem stated in his paper (Revue de la Faculté des Sciences de Université d'Istanbul (A) vol. 12 (1947)) for doubly connected domains $C$. Using the formulas of Muschelisvili and the theory of uniformization he shows that all solutions of this problem can be obtained by means of Weierstrass' functions $\sigma, \zeta, \theta$, corresponding to the periods $\omega, i \omega^{\prime}$ with $\omega>0, \omega^{\prime}>0$. Explicit formulas are given which furnish the totality of the solutions of the problem for the particular case when the stresses $\sigma_{x}, \sigma_{y}, \tau$ are finite everywhere in $C$ and on its boundary. (Received December 14, 1950.)

\section{4t. Raoul Bott and R. J. Duffin: The Wang algebra of networks.}

According to K. T. Wang, the determinant of Kirchhoff's equations is the "prod" uct" of any complete set of meshes of the network evaluated by the rules $r_{i}+r_{i}=0$ and $r_{i} \cdot r_{i}=0$. Each factor is the sum of the resistances $r_{i}$ in the mesh. More generally, let $D$ be the determinant of the quadratic form $\sum_{1}^{n} r_{i} x_{i}^{2}$ where the vector $\left(x_{1}, x_{2}, \cdots, x_{n}\right)$ is constrained to lie in a given $m$-dimensional subspace $K$ of a real vector space $V$. Then $D$ is a homogeneous multilinear form of degree $m$ with positive coefficients as a function of the parameters $r_{i}$ (see Bull. Amer. Math. Soc. Abstract 55-3-173). It is shown that $D$ is obtainable from the Grassmann product $(x \cdot y=-y \cdot x)$ for any complete set of $m$ vectors of $K$. The coefficients of $D$ are equal if and only if the vectors of $K$ having coordinates \pm 1 or 0 constitute an $m$-dimensional vector space under addition mod 2. For networks without transformers the constraint imposed by either one of Kirchhoff's laws has this property. The observation that the Wang algebra is simply the Grassmann algebra mod 2 then extends Wang's rule to its limit. (Received November 15, 1950.)

\section{Nathaniel Coburn: The independent scalars in isotropic turbu- lence.}

The independent scalars of the correlation tensors in isotropic turbulence are defined by the following three properties: (1) they must be the orthogonal components of the correlation tensors; (2) these scalars must be defined in such a manner that the correlation tensors satisfy the continuity relations identically; (3) the maximum number of such scalars must occur in each correlation tensor. In this paper, we furnish a method for constructing such scalars. The method is based upon the following procedure. First, one determines the maximum number of independent scalars associated with a given correlation tensor. Secondly, by a contraction process, a new tensor is constructed which possesses the maximum number of independent scalars. 
By forming the curl of this tensor, one obtains the desired tensor. The method is applied to: (1) the case of isotropic turbulence; (2) the case of axial symmetric turbulence. Finally, it is shown that our method generates all possible correlation tensors which depend linearly upon a maximum number of independent scalars and their derivatives and whose divergence vanishes identically. (Received July 28, 1950.)

\section{6t. David Gilbarg: Unsteady flows with finite closed wake.}

The free boundary problem for unsteady flows differs from the classical Helmholtz free boundary problem in that the boundary condition, flow speed $=|\operatorname{grad} \phi|$ $=$ const. on free streamlines, for the Helmholtz problem, is replaced by the condition (obtained from the nonstationary form of Bernoulli's equation) $\partial \phi / \partial t+|\operatorname{grad} \phi|^{2}$ $=c(t)$ on free streamlines, where $\phi$ equals the velocity potential. If unsteady plane flows past polygonal obstacles are considered, it is possible to derive in closed form the entire set of flows with finite closed wake (cavity) behind such obstacles. Standard methods of conformal mapping suffice. In the case of symmetric flow past a flat plate, von Kármán (Annali di Matematica Pura ed Applicata (4) vol. 29 (1949) pp. 247-249) has exhibited an example in which the wake is of constant shape. It is seen that this is a particular example of a class of flows with this property. (Received November 13, 1950.)

147. O. O. Pardee: An improperly-set boundary value problem in potential theory.

From the theory of electron flow the following problem is set. Given Cauchy conditions of potential and normal derivatives as continuous functions on an open surface, determine a solution in the large. No exact solution exists in general, but a solution satisfying the boundary conditions to an arbitrary degree of approximation is obtained for two dimensions, with suitable restrictions on the continuity of the boundary functions and on the smoothness of the boundary curve. The solution exists in any finite simply-connected region whose boundary is formed by the given (support) curve and a sufficiently smooth closing curve joining the end points of the support curve. A singularity is allowed at an arbitrarily chosen point on the closing curve. By solving a Neumann problem for this region, the normal derivative condition is satisfied exactly. To satisfy the remaining condition approximately, the region is mapped onto the upper half-plane, the support curve going into a finite portion of the $x$-axis and the singular point, to infinity. By means of an incomplete Fourier transform the approximate solution is obtained. Some special solutions are obtained for three dimensions. (Received November 13, 1950.)

148t. H. E. Salzer: Equally weighted quadrature formulas over semiinfinite and infinite intervals.

Equally weighted quadrature formulas of the type $\int_{a}^{b} F(x) f(x) d x=K \sum_{i=1}^{n} f\left(x_{i}\right)$ have been studied by Chebyshev and others for only finite values of $a$ and $b$. This present study is concerned with formulas of the type (1) $\int_{0}^{\infty} e^{-x} f(x) d x \doteq(1 / n) \sum_{i=1}^{n} f\left(x_{i}\right)$ and (2) $\int_{-\infty}^{\infty} e^{-x^{2}} f(x) d x=\left(\pi^{1 / 2} / n\right) \sum_{i=1}^{n} f\left(x_{i}\right)$ which are exact for $f(x)$ equal to I. any polynomial of degree $n$, or II. any polynomial of degree at most $m<n$ when the points $x_{i}$ are specified to all lie within the real intervals of integration. For I., the coefficients of the polynomials of which the $x_{i}$ are the zeros were obtained for $n \leqq 10$, in two different ways, and the values of $x_{i}$ (not all real for $10 \geqq n>2$ in (1) and for $10 \geqq n>3$ in (2)) were calculated to $7 D$. For II., it was proven that, in (1) $m=3$ requires $n \geqq 7$ and $m$ cannot exceed 3 even for $n$ as high as 14 , and in (2) $m=5$ requires $n \geqq 7$, with no 
possible increase in $m$ even for $n$ as high as 10 . The points $x_{i}$ for a formula in (1) where $n=7, m=3$, and a formula in (2) where $n=7, m=5$ were calculated to $12 D$ and $7 D$ respectively, together with checking factors to $10 S$ and $7 S$ respectively. (Received September 19, 1950.)

\section{C. B. Smith: An application of tensor methods to plates lam-} inated of orthotropic material.

Plates laminated from orthotropic layers, that is, layers that have two perpendicular axes of elastic symmetry in the plane of the layer, are usually constructed so that the adjacent layers have their axes of symmetry respectively parallel. This paper first discusses the elastic properties of a two-layer plate constructed with the axes of symmetry of the first layer inclined at any angle with the axes of symmetry of the second layer. It is shown that a plate of this type may be considered to be orthotropic. By using the formulas obtained for the two-layer case, plates with any even number of layers may be constructed (the adjacent layers will not necessarily have their axes of symmetry respectively parallel) which again may be considered to be orthotropic. Finally the results of this paper are applied to plates of plywood. (Received November 6,1950.)

\section{C. A. Truesdell: $A$ measure of vorticity.}

The local rotation in a continuous medium in motion is measured by the vorticity vector $w^{\rightarrow} \equiv \operatorname{curl} v \vec{v}, v$ being the velocity. Since $w^{\rightarrow}$ is not dimensionless, its numerical magnitude in any given motion has no individual significance, since it may be assigned any magnitude whatever by mere choice of units. In this paper an absolute measure of vorticity, $W \equiv w /\left(2 I I_{d}\right)^{1 / 2}$, where $I I_{d}$ is the second principal invariant of the rate of deformation tensor, is introduced and studied. All motions (with the exception of a uniform translation) are thus assigned a non-negative numerical degree of rotationality, varying from $W=0$ for an irrotational motion to $W=\infty$ for a rigid rotation. The class of motions for which $W=1$ is characterized; sufficient, but not necessary, is that the motion be slow. On the basis of the range of $W$, the Couette flows may be classified into five types, and it turns out that Rayleigh's criterion of stability for these flows (at infinite Reynolds number) may be put into the following form: a necessary and sufficient condition for stability is $W>1$ at all points. It is shown that for the Gerstner waves $W \leqq 1$, the value 1 being attained at the extreme possible amplitude. If $W \leqq 0.1$ it is necessary that the amplitude nowhere exceed $1.5 \%$ of the wave length. Thus Gerstner's waves are strongly rotational except for very small amplitudes. (Received October 26, 1950.)

\section{A. H. Van Tuyl: On the coefficients of capacity of two neighbor-} ing spheres.

Closed expressions are found for the coefficients of capacity of two neighboring spheres in the form of definite integrals whose integrands contain elliptic functions. Using these integral representations, series expansions are obtained which converge rapidly when the distance between the spheres is small. The expansions are used in the study of the charge density at the points of former contact when two charged tangent spheres are separated slightly. (Received November 13,1950.)

152. Alexander Weinstein: On dislocations in shafts under torsion.

The superposition of a uniform flow and of a flow generated by a vortex ring in a space of five dimensions yields the solution of the problem of torsion of a nearly 
cylindrical shaft of revolution with an interior crack bounded by a circular line of radius $b$. The potential of the vortex line is given in terms of Bessel functions by the formula const. $\int_{0}^{\infty} \exp (-|x| t) J_{1}(y t) J_{2}(b t) d t$. This formula follows from the results given by A. Weinstein, Proceedings of the seventh international congress for applied mechanics, 1948, pp. 108-119. In this formula $x$ and $y$ denote cylindrical coordinates in the meridian plane. (Received October 19, 1950.)

\section{GEOMETRY}

\section{3t. John DeCicco: Commutators of turns, slides, and dilatations} upon a surface.

New proofs and extensions of certain theorems of Kasner concerning infinitesimal lineal element transformations are given. The commutators between the symbols $U_{1} F, U_{2} F, U_{3} F$ of the three one-parameter groups of turns, slides, dilatations upon a surface $\Sigma$ are $\left(U_{1}, U_{2}\right) F=U_{3} F ;\left(U_{2}, U_{3}\right) F=K U_{1} F ;\left(U_{3}, U_{1}\right) F=U_{2} F$, where $K$ is the Gaussian curvature of $\Sigma$. The turns and slides form a group of a finite number of parameters if and only if $\Sigma$ is of constant Gaussian curvature. If $K=$ const., then this is the whirl group of three parameters, which contains the dilatations as a subgroup. Two infinitesimal contact transformations, neither of which are point transformations, such that they possess the same transversality law and the commutator is a point transformation, are two infinitesimal dilatations of two conformally equivalent surfaces. Two infinitesimal contact transformations possess the same linear involutorial law of transversals if and only if they are infinitesimal dilatations of two conformally equivalent surfaces. (Received November 27, 1950.)

154t. John DeCicco: Conservative physical systems of curves upon a surface.

A system $S_{k}$ of $\infty^{3}$ curves in a given field of force upon a surface $\Sigma$ consists of curves along which a constrained motion is possible such that the pressure $P$ is proportional to the normal component $N$ of the force vector. Thus $P=k N$ where $k(\neq-1)$ is the constant factor of proportionality. A conservative $S_{k}$ is a physical system in a conservative field of force. The special cases $k=0,1,-2, \infty$ define the $\infty^{3}$ dynamical trajectories, catenaries, brachistochrones, velocity curves, respectively. The Lagrangian and Hamiltonian equations for a conservative $S_{k}$ are obtained. A conservative $S_{k}$, where $k \neq \infty$, can be separated into $\infty^{1}$ natural families $S_{k}(E)$, according to the value of the energy constant $E$. The natural family $S_{k}(E)$ consists of $\infty^{2}$ geodesics of a surface, and hence forms the trajectories of the one-parameter group of dilatations of this surface. The infinitesimal dilatation of this group is an extension of the infinitesimal contact transformation of mechanics, studied extensively by Lie, Vessiot, Kasner. Similar results are obtained for a velocity system $S_{\infty}$. (Received November $27,1950$. .)

\section{Patrick Du Val: Surfaces whose canonical systems are hyper-} elliptic.

If the irreducible canonical system on a regular algebraic surface of genera $p_{y}=p_{a}=p, p^{(1)}=n+1$, consists of hyperelliptic curves, it has $n-2 p+4$ simple base points on a model free from exceptional curves. The canonical model is a double normal rational ruled surface of order $p-2$ on which the base points appear as lines $\lambda$, which are constituents of the branch curve (for $p=3$ a double plane, for $p=6, n=8$ alternatively a double Veronese surface). There is a residual branch curve $f$ of order 
$n+2 p$, which has a $(k+2)$ ple point at every point common to $k$ of the lines $\lambda$, and further triple points at general points on these lines, sufficient on each to reduce its virtual grade to -2 . These points are base points of the bicanonical system, so that on the bicanonical model the base points of the canonical system are isolated branch points at conical nodes. Generally the lines $\lambda$ are generators of the ruled surface (or belong to a pencil for $n=3$ ) and the residual branch curve meets each generator in six points and has two triple points in each line $\lambda$. There are a small number of exceptional cases for $p \leqq 6, n \leqq 9$. (Received November 13,1950.)

\section{A. J. Hoffman: Cyclic affine planes. Preliminary report.}

Consider a finite affine plane that admits a collineation which leaves one point fixed and permutes the others cyclically. As R. C. Bose has indicated (J. Indian Math. Soc. (2) vol. 6 (1942)), such a "cyclic affine plane" with $n$ points on a line can be constructed from a difference set of $n$ integers $\left(\bmod n^{2}-1 ; n+1\right)$ (in the notation of Chowla, J. Indian Math. Soc. (2) vol. 9 (1945)), and conversely. The author makes additional comments about cyclic affine planes, in analogy with M. Hall's discussion of cyclic projective planes (Duke Math J. vol. 14 (1947)). The most interesting is that $p \mid n$ implies $p$ is a "multiplier" of the difference set. This extends a theorem of Chowla (loc. cit.). (Received November 14, 1950.)

157. Benjamin Ernest Mitchell: Synthetic development of Laguerre's representation of the imaginary point.

The complex complete quadrangle developed from four imaginary points conjugate in pairs has a real diagonal triangle. The fate of this diagonal triangle when one of the conjugate pairs become the circular points at infinity determines the Laguerre representation. Four of the six sides become isotropics. One becomes the line at infinity. The sixth, real and carrier of the second conjugate point-pair, remains finite. One of the diagonal points goes to infinity. The other two take positions symmetric to the line of the finite conjugate point-pair, and with them constitute a parti-real square. They, when ordered, are the real representation of the finite conjugate imaginary point-pair. (Received November 9, 1950.)

158t. Benjamin Ernest Mitchell: Relation of two lines in the complex plane.

Since a line in the complex plane is two-dimensional in points, two such lines may be expected to have relationship in excess of their common point. And such they have. Theorem: Any two lines in the complex plane share a common point and a conjugate point-pair. The "pictures" (Study) of the four points involved when the conjugates of the lines are also taken into consideration determine the type of lines involved. The special cases of Kreutzlage, Trapezlage, and Lage isometrich (Study) are considered. (Received November 9, 1950.)

\section{9t. Samuel Verblunsky: On the shortest path through a number of} points.

It is proved that, given $\nu$ points $P_{i}\left(x_{i}, y_{i}\right)$ in the strip $0 \leqq y \leqq 1$ with $x_{1} \leqq x_{2} \leqq \cdots \cdot$ $\leqq x_{\nu}$, there is a path whose first point is $P_{1}$, whose last point is $P_{j}$ say, which contains the $\nu$ points, and whose length does not exceed $x_{j}-x_{1}+.7 \nu$. Here, .7 cannot be replaced by a number less than $3^{-1 / 2}$. It is deduced that, given $n$ points in a unit square, there is a path containing them of length less than $2+(2.8 n)^{1 / 2}$. (Received November $6,1950$. 


\section{Statistics AND Probability}

\section{0t. K. L. Chung: An extension of renewal theory.}

Let $\left\{X_{n}\right\}, n=1,2, \cdots$, be a sequence of independent random variables having the common distribution function $F(x)$ and let $S_{n}=\sum_{1}^{n} X_{k}$. Suppose that the mean of $F(x)$ is a finite positive number $m$. If $F(x)$ is of the aperiodic lattice type, then $\lim \sum_{1}^{\infty} P\left(S_{n}=x\right)$ is equal to $1 / m$ or 0 according as $x \rightarrow+\infty$ or $x \rightarrow-\infty$ on the lattice. If $F(x)$ contains a nonvanishing absolutely continuous part, then $\lim \sum_{1}^{\infty} P\left(x<S_{n}\right.$ $\leqq x+h)$ is equal to $h / m$ or 0 according as $x \rightarrow+\infty$ or $x \rightarrow-\infty$. These theorems extend theorems of Erdös, Feller, and Pollard and of Doob and Blackwell on renewal theory. The method of proof is new. (Received November 14, 1950.)

161t. F. G. Tricomi: The probable length of the resultant of $n$ random unit vectors.

In a problem of biology it was required to determine the average $l_{n}$ of the length $r$ indicated in the title, which is a continuous random variable in the range $(0, n)$. It was possible for the author to determine rigorously the probability density $\phi_{n}(r)$ of $r$, which is given by $(1 / 2) r\left\{\tau_{n-1}[(n-r) / 2]-\tau_{n-1}[(n+r) / 2]\right\}$ where $\tau_{n}(x)$ is another probability density already determined by the author in 1932 (Jber. Deutschen Math. Verein. vol. 42, pp. 174-179). Actually $\tau_{n}(x)$ is the probability density of the sum of $n$ independent random variables, each with the constant probability density one in the range $(0,1)$. Moreover, an asymptotic representation of $\phi_{n}(r)$ is found (a distribution of the type $\left.C r^{2} \exp \left[-3 r^{2} / 2 n\right]\right)$ and thereafter the asymptotic expression $2(2 n / 3 \pi)^{1 / 2}+O\left(n^{-1 / 2}\right)$ of the average is obtained. (Received November 9,1950 .)

\section{Topology}

\section{2t. W. R. Baum: The group of null-spherods.}

A combinatorial null-spherod in a complex $K$ is a simplicial mapping of an oriented $n$-sphere into $K$ which has degree 0 in each $n$-simplex of $K$. Two null-spherods $s_{1}, s_{2}$ are $\nu$-homotopic if there is a combinatorial deformation of $s_{1}$ into $s_{2}$ such that all spherods between them are also null-spherods. Using these notions instead of ordinary sphere images and homotopies, define, in analogy to the Hurewicz groups, new groups $\Psi^{n}(K)$. Similar groups can be defined for continuous mappings and continuous deformations; they are topological invariants of $K . \Psi^{n}(K)$ is isomorphic to the factorgroup $\Gamma^{n}\left(K^{n}\right) / \mathrm{P}_{0}^{n}\left(K, K^{n}\right)$, where $\Gamma^{n}\left(K^{n}\right)$ and $\mathrm{P}_{0}^{n}\left(K, K^{n}\right)$ are subgroups of the Hurewicz homotopy group $\pi_{n}\left(K^{n}\right)\left(K^{n}=n\right.$-dimensional skeleton of $K$ ) introduced by H. Hopf (Comment. Math. Helv. vol. 17 (1945) pp. 307-326). $\Gamma^{n}\left(K^{n}\right)$ is that subgroup of $\pi_{n}\left(K^{n}\right)$ whose elements are represented by $n$-spherods in $K^{n}$ which are 0 in the sense of homology. The elements of $\pi_{n}\left(K^{n}\right)$ which are null-homotopic in $K$ form a group $\mathrm{P}^{n}\left(K, K^{n}\right)$. Then $\mathrm{P}_{0}^{n}\left(K, K^{n}\right)$ is the subgroup of $\mathrm{P}^{n}\left(K, K^{n}\right)$ which is generated by all differences $\rho-\rho^{\prime}$ where the elements $\rho, \rho^{\prime} \in \mathrm{P}^{n}\left(K, K^{n}\right)$ are free-homotopic on $K^{n}$. (Received November 13, 1950.)

\section{3t. A. L. Blakers and W. S. Massey: On the homotopy groups of} spheres.

In this paper the definition of the $J$-homomorphism of G. W. Whitehead (Ann. of Math. vol. $51(1950)$ p. 214$)$ is extended to give a homomorphism $J_{1}: \pi_{p}\left(R_{k-1}, R_{k-2}\right)$ $\rightarrow \pi_{p+k}\left(S^{k} ; E_{+}^{k}, E_{-}^{k}\right)$, and a homomorphism of the homotopy sequence of the pair $\left(R_{k-1}, R_{k-2}\right)$ into one of the homotopy sequences of the triad $\left(S^{k} ; E_{+}^{k}, E_{-}^{k}\right)$. A "Hopf 
homomorphism" $H_{1}: \pi_{n}\left(S^{r} ; E_{+}^{r}, E_{-}^{r}\right) \rightarrow \pi_{n+1}\left(S^{2 r}\right)$ is defined for all $n, r . \kappa_{*}: \pi_{p}\left(R_{k-1}, R_{k-2}\right)$ $\approx \pi_{p}\left(S^{k}\right)$ is the isomorphism induced by the fiber map of $R_{k-1}$ on $S^{k}$ and $E^{(k+1)}$ denotes the $(k+1)$-fold suspension. The commutativity relation $H_{1} J_{1}(\alpha)=E^{(k+1)} \kappa_{*}(\alpha)$, $\alpha \in \pi_{p}\left(R_{k-1}, R_{k-2}\right)$ is proved. Thus if $\pi_{p}\left(S^{k}\right)$ has an element whose successive suspensions are nontrivial, then $\pi_{p+k}\left(S^{k} ; E_{+}^{k}, E_{-}^{k}\right)$ is nontrivial. Hence either $\pi_{p+k}\left(S^{k}\right) \neq 0$ or $\pi_{p+k-2}\left(S^{k-1}\right) \neq 0$. This result overlaps with that of Everett Pitcher (Bull. Amer. Math. Soc. Abstract 56-1-38). (Received August 30, 1950.)

\section{4t. A. L. Blakers and W. S. Massey: Homotopy groups of triads. III.}

Let $(X ; A, B)$ be a triad such that every point of $X$ belongs to the interior of $A$ or the interior of $B$, and $A, B$, and $A \cap B$ are all arcwise connected. Theorem: If $(A, A \cap B)$ is $m$-connected, $m \geqq 2$, and $(B, A \cap B)$ is $n$-connected, $n \geqq 2$, then the triad $(X ; A, B)$ is $m+n$ connected. This theorem is still true if $m=1$, or $n=1$, provided $A \cap B$ is simply connected. Corollary 1 : Let $(X, A)$ be a triangulable pair such that $(X, A)$ is $m$-connected, $m \geqq 1$, and $A$ is $n$-connected, $n>1$. Let $\widetilde{X}$ be the space obtained by identifying all of $A$ to a single point, $x_{0}$. Let $\phi:(X, A) \rightarrow\left(\widetilde{X}, x_{0}\right)$ denote the identification map, and $\phi_{p}: \pi_{p}(X, A) \rightarrow \pi_{p}\left(\widetilde{X}, x_{0}\right)$ the homomorphisms induced by $\phi$. Then $\phi_{p}$ is an isomorphism onto for $p \leqq m+n$, and is a homomorphism onto for $p=m+n+1$. Corollary 2: Let $X$ be a simplicial complex and $A$ and $B$ subcomplexes such that $X=A \cup B$, and $A, B$, and $A \cap B$ are all connected. Assume that $(A, A \cap B)$ is $m$-connected, $m \geqq 1,(B, A \cap B)$ is $n$-connected, $n \geqq 1$, and $A \cap B$ is $r$-connected, $r>1$. Then the injections $\pi_{p}(A, A \cap B) \rightarrow \pi_{p}(X, B)$ and $\pi_{q}(B, A \cap B) \rightarrow \pi_{q}(X, A)$ are isomorphisms into for $p \leqq m+r$ and $q \leqq n+r$ respectively. (Received November 13, 1950.)

165t. A. L. Blakers and W. S. Massey: Generalized Whitehead products. II.

Let $(X ; A, B)$ be a triad. A new product is defined which associates with elements $\alpha \in \pi_{p}(B, A \cap B)$ and $\beta \in \pi_{q}(A, A \cap B)$ an element $[\alpha, \beta]$ of $\pi_{r}(X ; A, B), r=p+q-1$. This product is bilinear if $p$ and $q$ are greater than 2 , and satisfies the commutation rule $[\alpha, \beta](-1)^{p q}[\beta, \alpha]$. If $\partial_{+}: \pi_{r}(X ; A, B) \rightarrow \pi_{r-1}(A, A \cap B)$ and $\partial_{-}: \pi_{r}(X ; A, B)$ $\rightarrow \pi_{r-1}(B, A \cap B)$ denote the boundary operators of the triad $(X ; A, B)$, and $\partial_{1}: \pi_{q}(A, A \cap B) \rightarrow \pi_{q-1}(A \cap B), \partial_{2}: \pi_{p}(B, A \cap B) \rightarrow \pi_{p-1}(A \cap B)$ are boundary operators of the pairs $(A, A \cap B)$ and $(B, A \cap B)$, then $\partial_{+}[\alpha, \beta]=-\left[\partial_{2} \alpha, \beta\right]$, and $\partial_{-}[\alpha, \beta]$ $=(-1)^{p-1}\left[\alpha, \partial_{1} \beta\right]$. Various other relations between this new product and the products previously defined by J. H. C. Whitehead and the authors are established. (Received November 13, 1950.)

166t. A. L. Blakers and W.S. Massey: On the groups $\pi_{p}\left(S^{n} ; E_{+}^{n}, E_{-}^{n}\right)$.

Let $S^{n}$ be an $n$-sphere, $E_{+}^{n}$ and $E_{-}^{n}$ the upper and lower caps, and $S^{n-1}=E_{+}^{n} \cap E_{-}^{n}$ the equator. Choose a generator $\iota \in \pi_{n}\left(E_{-}^{n}, S^{n-1}\right)$. Define a homomorphism $\theta_{p}$ : $\pi_{r}\left(E_{-}^{n}, S^{n-1}\right) \rightarrow \pi_{p}\left(S^{n} ; E_{+}^{n}, E_{-}^{n}\right)$, where $r=p-n+1$, by $\theta_{p}(\alpha)=[\alpha, \iota]$ for any $\alpha \in \pi_{r}\left(E_{-}^{n}\right.$, $\left.S^{n-1}\right)$. Here $[\alpha, \iota]$ denotes the generalized Whitehead product defined in the previous abstract. Let $\phi:\left(S^{n} ; E_{+}^{n}, E_{-}^{n}\right) \rightarrow\left(S_{1}^{n} \bigvee S_{2}^{n} ; S_{1}^{n}, S_{2}^{n}\right)$ be a map which identifies all of the equator $S^{n-1}$ to a single point, $x_{0} \in S_{1}^{n} \bigvee S_{2}^{n}$. The space $S_{1}^{n} \bigvee S_{2}^{n}$ consists of two spheres with a single point in common. Let $\phi_{p}: \pi_{p}\left(S^{n} ; E_{+}^{n}, E_{-}^{n}\right) \rightarrow \pi_{p}\left(S_{1}^{n} \bigvee S_{2}^{n} ; S_{1}^{n}, S_{2}^{n}\right)$ denote the homomorphism induced by $\phi$. Theorem: If $p<3 n-3$, then $\phi_{p} \theta_{p}$ is an isomorphism onto; if $p=3 n-3$, then $\phi_{p} \theta_{p}$ is a homomorphism onto. Corollary $1:$ If $p<3 n-3$, then $\pi_{p}\left(S^{n} ; E_{+}^{n}, E_{-}^{n}\right)$ has a direct summand isomorphic to $\pi_{p}\left(S^{2 n-1}\right)$. Corollary 2 : If $\pi_{3 n-3}\left(S^{2 n-1}\right) \neq 0$, then $\pi_{3 n-3}\left(S^{n} ; E_{+}^{n}, E_{-}^{n}\right) \neq 0$. Corollary 3: The following groups are 
not zero: $\pi_{2 n}\left(S^{n} ; E_{+}^{n}, E_{-}^{n}\right), n \geqq 3 ; \pi_{2 n+1}\left(S^{n} ; E_{+}^{n}, E_{-}^{n}\right), n \geqq 4 ; \pi_{2 n+2}\left(S^{n} ; E_{+}^{n}, E_{-}^{n}\right), n \geqq 5$. (Received November 13, 1950.)

167. A. L. Blakers: Geometric proof of an algebraic theorem of Eilenberg-MacLane.

S. Eilenberg and S. MacLane (Proc. Nat. Acad. Sci. U.S.A. vol. 36 (1950) p. 443) have proved the cohomology equivalent of the following algebraic theorem: The suspension homomorphism $S: H_{q}(K(\pi, n), G) \rightarrow H_{q+1}(K(\pi, n+1), G)$ is an isomorphism onto for $q<2 n$, and is onto for $q=2 n$. In the present paper this result is deduced as a corollary of a theorem about the homotopy groups of certain triads, and consequently the proof is rather geometric. Let $K$ be a complex with $\pi_{i}(K)=0, i<n, n<i<m$, $m>2 n, n>1$, and with $\pi_{n}(K)=\pi$. (See J. H. C. Whitehead, Ann. of Math. vol. 50 (1949) p. 261). Let $\widetilde{K}$ denote the join of $K$ with a pair of points. Then it follows from a general theorem about triad homotopy groups (Bull. Amer. Math. Soc. Abstract 57-2-162) that $\pi_{i}(\widetilde{K})=0, i<n+1, n+1<i \leqq 2 n$, while $\pi_{n+1}(\widetilde{K}) \approx \pi$. It now follows (see Eilenberg-MacLane, Ann. of Math. vol. 46 (1945) p. 508) that the homomorphism $k: H_{q}(K, G) \rightarrow H_{q}(K(\pi, n), G)$ is an isomorphism onto for $q \leqq m$, while the corresponding homomorphism $\widetilde{k}: H_{q}(\widetilde{K}, G) \rightarrow H_{q}(K(\pi, m+1), G)$ is an isomorphism onto for $q \leqq 2 n$ and is onto when $g=2 n+1$. Moreover, the homology suspension homomorphism $h: H_{q}(K, G) \rightarrow H_{q+1}(\tilde{K}, G)$ is an isomorphism onto for all dimensions, and the theorem now follows from the commutativity relation $\widetilde{k} h=S k$. (Received November 13, 1950.)

168t. Raoul Bott: $A$ set of new combinatorial invariants for cell complexes. Preliminary report.

Let $K$ be a finite $n$-dimensional cell complex, with the $n$ cells $\sigma_{i}\{i=1,2, \cdots, \lambda\}$. If $S$ is a subset of the first $\lambda$ integers, denote by $K_{s}$ the complex derived from $K$ by deleting all $n$ cells $\sigma_{i}$ with $i \notin S$. $\beta^{\alpha}\left(K_{\varepsilon}\right)$ shall stand for the $\alpha$ th Betti number of $K_{s}$, $|s|$ shall be the number of elements in the set $S$. Set $p_{\mu}=\sum_{\beta^{n}\left(K_{s}\right)-\mu}(-)^{|s|} ; q_{\mu}$ $=\sum_{\beta^{n}\left(K_{s}\right)-\mu}\left((-1)^{1 / 2}\right)^{|s|-\beta^{n-1}\left(K_{s}\right)}$. Then $p_{\mu}^{2}, q_{\mu}^{4}$ are invariants of $K$ under subdivision; however they are not homotopy invariants. (Received November 16, 1950.)

169. K. T. Chen: On isotopic invariants of links.

An $n$-link $L$ is the union of $n$ disjoint simple closed curves in a euclidean 3-space $E$. Each of the closed curves is called a component of $L$. A link with polygonal components is said to be polygonal. An isotopy $\phi: L \times I \rightarrow E$ is regular if each link $\phi_{t_{0}}(L)$, $0 \leqq t_{0} \leqq 1$, is polygonal with respect to some coordinate system of $E$, and, given $\epsilon>0$, there is $\delta>0$ such that, for each $t,\left|t-t_{0}\right|<\delta$, there is a homeomorphism $\tau: E \rightarrow E$ with $\tau \phi_{t}(L)$ being polygonal and $\left|\tau \phi_{t}(p)-\phi_{t_{0}}(p)\right|<\epsilon, p \in L$, with respect to the same coordinate system. Let $G$ and $G^{\prime}$ be the fundamental groups of $E-\phi_{0}(L)$ and $E-\phi_{1}(L)$ respectively. Then $G / G_{d}$ and $G^{\prime} / G_{d}^{\prime}$ are isomorphic $(d=1,2, \cdots)$, where $G_{d}$ and $G_{d}^{\prime}$ are the $d$ th lower central commutator subgroups of $G$ and $G^{\prime}$ respectively. However groups $G$ and $G^{\prime}$ are, in general, not isomorphic. Under a regular isotopy, each finitely generated abelian group $G_{d} / G_{d+1}$ is therefore invariant and yields numerical invariants. (Received November 13,1950 .)

\section{0t. D. O. Ellis: On separable metric spaces.}

Let $M$ be a separable metric space. Let $M^{*}$ be a subset dense in $M$ which is at most countable. We assume that $M^{*}$ is fixed as to sequentialization and let its elements be $q_{1}, q_{2}, \ldots$. It is easily shown (and well known) that $M^{*}$ forms a metric 
base for $M$. We attach to each point of $M$ as metric coördinates the distances $p q_{1}, p q_{2}, \cdots$. The results of this note are: 1. The mapping of $M$ into Fréchet's space $E_{\omega}$ defined by corresponding coördinates is biuniform and continuous and is a homeomorphism when $M$ is compact; 2 . The coördinate convergence in $M$ is equivalent to the original metric convergence; 3 . If $p$ and $q$ are points of $M$ with coördinates $\left\{x_{i}\right\}$ and $\left\{y_{i}\right\}$, respectively, then $p q=$ g.l.b.i $\left(x_{i}+y_{i}\right)=1 . u . b_{i}\left|x_{i}-y_{i}\right|$. Two unsolved problems are also proposed in the note. (Received August 11, 1950.)

171. D. O. Ellis: On strict metricity in metric spaces.

A $\gamma$-metric space is a semimetric space satisfying $\left(^{*}\right) x y+y z \geqq \gamma x z$ for $x \neq y \neq z \neq x$. If $M$ is a $\gamma$-metric space for $\gamma>1, M$ is called a more than metric space. A metric space not satisfying $\left(^{*}\right)$ for any $\gamma>1$ is called strictly metric. If $M$ is a metric space, the curvature ray, $K(M)$, of $M$ is the set of all $\gamma \cdot \epsilon[0, \infty]$ satisf ying $\gamma=\min ((x y+y z) / x z$, $(x y+x z) / y z,(x z+y x) / x y)$ for $x, y, z \in M, x \neq y \neq z \neq x$. The principal results of the present paper are: 1. Every more than metric space is discrete in its metric topology; 2. For a metric space of more than two distinct points not containing any linear triples to be strictly metric, it is necessary and sufficient that $1 \in K^{\prime}(M)$; 3 . For a metric space to be strictly metric it is sufficient that either the space contain a linear triple or that the space contain at least one accumulation point in its metric topology. (Received August 3, 1950.)

172t. E. E. Floyd: Some retraction properties of the orbit decomposition spaces of periodic maps.

Let $X$ be a finite-dimensional locally compact metric space and let $T$ be a periodic map on $X$. Let $X^{*}$ denote the orbit decomposition space of $X, T$. Then if $X$ is an absolute neighborhood retract (absolute retract), so also is $X^{*}$. (Received November 13, 1950.)

\section{3t. E. E. Floyd: Examples of fixed point sets of periodic maps.}

Examples are constructed to prove that if $G$ is any nontrivial abelian group, there exists a finite complex $X$ and a simplicial map $T$ of $X$ onto $X$ of some prime period such that $X$ is homologically trivial over $G$ while the fixed point set $L$ of $T$ is not homologically trivial over $X$. Similarly there exists an $X$ and a $T$ such that $X$ is a homological sphere over $G$ while $L$ is not. These results answer a question of P. A. Smith (see page. 372 of his appendix in Lefschetz, Algebraic topology). (Received November 13, 1950.)

174. F. B. Jones: Certain homogeneous, unicoherent, indecomposable continua.

It is shown in a metric space that: Every homogeneous, unicoherent, compact continuum is indecomposable. It follows that in the plane: Every homogeneous, bounded continuum which does not separate the plane is indecomposable. Is every such continuum a pseudo-arc? (Received November 22, 1950.)

175t. Saunders MacLane and J. H. C. Whitehead: The complete 3-homotopy type of a complex.

In a recent note in Proc. Nat. Acad. Sci. U.S.A. (vol. 36 (1950) pp. 41-47) the authors have shown that the three-type of a CW complex can be described by the homotopy groups $\Pi_{1}$ and $\Pi_{2}$ of $K$, the operators of $\Pi_{1}$ on $\Pi_{2}$, and the homotopy obstruc- 
tion cohomology class $k$ in $H^{3}\left(\Pi_{1}, \Pi_{2}\right)$. We now consider the corresponding problem of the classification of maps of one such complex $K$ into another. By making special choices in the construction of $K$, we obtain a specific cocycle $l$ in $Z^{3}\left(\Pi_{1}, \Pi_{2}\right)$. The 3homotopy classification of maps can then be formulated combinationally in terms of the actions of these maps on the cocycles $l$. (Received November 14,1950.)

\section{6t. Deane Montgomery and Leo Zippin: Four-dimensional} groups.

This paper shows that every four-dimensional locally compact separable metric locally connected group is a Lie group. The proof makes use of the existence of a closed two-dimensional subgroup which has recently been shown by the authors. (Received November 13, 1950.)

\section{G. D. Mostow: On the topology of noncompact Klein spaces. I.}

A new representation theorem for positive definite symmetric matrices is proved and with its help a simplified model for certain Klein spaces is obtained. Theorem. Let $S$ denote the space of all real symmetric $n \times n$ matrices, let $E$ be a linear subspace of $S$, let $\dot{F}=\{X \mid X \in S, \operatorname{Tr} X Y=0$, all $Y \in E\}$, and let $P, E, F$ denote $\exp S$, exp $E$, $\exp F$ respectively. Assume that for all $x, y \in E, x y x \in E$. Then for any $p \in P, p=e f e$ uniquely and continuously with $e \in E$ and $f \in F$. Let $K$ be a compact Lie group, $K^{\prime}$ a closed subgroup of $K, F$ a euclidean space, and $\lambda$ a continuous homomorphism of $K^{\prime}$ into the orthogonal group on $F$. Let $f^{k}=\lambda(k)(f)$ if $k \in K^{\prime}$ and $f \in F$. If $(x, f) \in K \times F$, define $C_{x, f}=\left\{(x k, f) \mid\right.$ all $\left.k \in K^{\prime}\right\}$. Definition. $\left(K, K^{\prime}, F, \lambda\right)$ is the base space obtained upon taking the $C_{x, f}$ as fibers in $K \times F$. Theorem. Any Klein space whose isotropy subgroup is connected and semisimple is topologically $\left(K, K^{\prime}, F, \lambda\right)$ where $K, K^{\prime}, F, \lambda$ are suitably determined. If $\lambda$ can be extended to a continuous mapping of $K$ into the group of auto-homeomorphisms of $F$ such that $\lambda(x k)=\lambda(x) \cdot k, x \in K, k \in K^{\prime}$, then $\left(K, K^{\prime}, F, \lambda\right) \cong K / K^{\prime} \times F$. This is the case if $K / K^{\prime}$ admits a continuous cross-section n $K$. ( Received December 20, 1950.)

\section{8t. R. L. Taylor: The extendibility of a crossed module.}

J. H. C. Whitehead has defined the concept of a crossed module [Bull. Amer. Math. Soc. vol. 55 (1949) pp. 454-496], and has shown that crossed modules appear in the homotopy systems of complexes. MacLane and Whitehead have defined the obstruction of a crossed module [Proc. Nat. Acad. Sci. U.S.A. vol. 36 (1950) pp. 41-48] and used it to characterize the 3-type of a complex. The present paper is the first in a series showing the role played by crossed modules in the theory of universal covering groups. The concept of an extension of a crossed module is defined, and it is proved that a crossed module is extendible if and only if its obstruction vanishes. Other necessary and sufficient conditions for the extendibility of special kinds of crossed modules are given, in terms of the cohomology theory of groups. (Received November 16,1950.)

\section{9t. R. L. Taylor: Universal coverings of a nonconnected group.}

A covering of the topological group $G$ is a topological group $G^{*}$ together with a homomorphism $p$ of $G^{*}$ onto $G$ such that for each arcwise component $M$ of $G ; p^{-1}(M)$ is arcwise connected, and $p / p^{-1}(M)$ is a fibre mapping with discrete fibre. Such a covering is universal if the arcwise components of $G^{*}$ are simply connected. A nonconnected topological group $G$ may fail to admit a universal covering, even if $G$ is a compact 1-dimensional Lie group. Let $G$ be a topological group such that $N$, the arcwise 
component of the identity, has sufficient local properties for the existence of a universal covering $N^{*}$ of $N$. It is proved that $N^{*}$ forms a crossed module over $G$, and that the universal coverings of $G$ are in a natural 1-1 correspondence with the extensions of the crossed module $\left(N^{*}, G\right)$. Hence $G$ admits a universal covering if and only if the obstruction of the crossed module $\left(N^{*}, G\right)$ vanishes (R. L. Taylor, The extendibility of a crossed module, Bull. Amer. Math. Soc. Abstract 57-2-178). This obstruction may be regarded as an element of $H^{3}\left[G / N, \pi_{1}(N)\right]$; it is an invariant of $G$. The results are generalized in a subsequent paper ( $R$. L. Taylor, Universal coverings of a ringoid, Bull. Amer. Math. Soc. Abstract 57-2-180). (Received November 16, 1950.)

180t. R. L. Taylor: Universal coverings of a ringoid.

A ringoid is an additive group $P$ (not necessarily abelian) together with an operation of multiplication in $P$, satisfying the axioms for a groupoid, such that if the products $a b$ and $c d$ are defined, then the product $(a+c)(b+d)$ is defined, and $(a+c)$ $\cdot(b+d)=a b+c d$. An example is the set of all strict homotopy classes of paths in an additive topological group ("strict" meaning "end points fixed"). It is proved that the concept of a ringoid is equivalent to that of an additive group $P$ together with a subgroup $E$ and an ordered pair of retraction-homomorphisms $L, R$ of $P$ onto $E$, such that every element of $L^{-1}(0)$ commutes with every element of $R^{-1}(0)$. From every ringoid $P$ a crossed module $M(P)$ is constructed in a natural way. The concepts of covering and universal covering of a ringoid are defined algebraically, and it is proved that the universal coverings of a ringoid $P$ are in a natural 1-1 correspondence with the extensions of the crossed module $M(P)$. This permits a generalization of the results of a previous paper (R. L. Taylor, Universal coverings of a nonconnected group, Bull. Amer. Math. Soc. Abstract 57-2-179) to the case of a topological group $G$ of which no local properties are assumed. (Received November 16, 1950.)

\section{1t. R. L. Taylor: The classification of fibre bundles. I.}

Let $B$ be a topological space, and $G$ a topological group. Let $B$ be coverable by two closed sets $M_{1}$ and $M_{2}$, each contractible in $B$, whose intersection $M_{12}$ is contractible in $M_{1}$ and in $M_{2}$, and is a neighborhood retract of $B$. Assume also that every homotopically trivial map defined on $M_{12}$ is extendible to $B$ (for example, let $B$ be metric separable and $M_{12}$ a compact ANR). Let $N$ be the arcwise component of the identity in $G$, and let $M_{12}^{\prime}$ be an arbitrary arcwise component of $M_{12}$. Theorem: The abstract fibre bundles over $B$ in $G$ are in 1-1 correspondence with the conjugacy classes (under $G / N)$ of all homotopy classes of maps of the pair $\left(M_{12}, M_{12}^{\prime}\right)$ into the pair $(G, N)$. Feldbau's classification of bundles over a sphere [C. R. Acad. Sci. Paris vol. 208 (1939) pp. 1621-1623] and Steenrod's classification of bundles over a 1-complex [lectures, University of Michigan, 1947] are special cases of this theorem. (The suspension of any compactum may be covered in the manner described above.) Some corollaries on the homotopy classification of mappings are given. (Received November 16,1950.)

\section{2t. R. L. Taylor: The classification of fibre bundles. II.}

This paper develops a method of classifying fibre bundles without using universal bundles. The method provides new results, which may be combined with the universal bundle theory to yield corollaries on the homotopy classification of mappings. Let $B$ be a topological space, and $G$ a topological group. A covering $C$ of $B$ is admissible if it satisfies certain conditions resembling those of the previous paper, but without restriction on the number of sets used. A homotopy cocycle on $C$ in $G$ consists of a homotopy class of maps of $M_{i} \cap M_{i}$ into $G$ for each ordered pair $M_{i}, M_{j}$ of sets in $C$; 
homotopies must be stationary throughout the intersection of any 3 distinct sets, and an algebraic condition of the form $H_{i j} H_{j k}=H_{i k}$ is required. Equivalence of homotopy cocycles is defined by a kind of conjugation by classes of homotopically trivial maps. Theorem: If $C$ is an admissible covering of $B$, the abstract bundles over $B$ in $G$ are in 1-1 correspondence with the equivalence classes of homotopy cocycles on $C$ in $G$. The main theorem of the preceding paper is an easy corollary. (Received November 16, 1950.)

\section{3t. R. L. Taylor: The classification of fibre bundles. III.}

The technique developed in the previous paper is applied here to the classification of all fibre bundles over a compact 2-manifold $B$, in an arbitrary topological group $G$. Complete results are obtained in terms of the ringoid $P(G)$ of all strict homotopy classes of paths in $G$. If $G$ admits an algebraic universal covering $G^{*}$ (R. L. Taylor, Universal coverings of a ringoid, Bull. Amer. Math. Soc. Abstract 57-2-180), the results can be stated in terms of $G^{*}$ instead of $P(G)$. Special cases: (1) If the center of $G$ meets every arcwise component of $G$, the bundles are in 1-1 correspondence with the direct sum $\pi_{1}(N)+\operatorname{Hom}\left[\pi_{1}(B), G / N\right]$ or $\pi_{1}(N) / 2 \pi_{1}(N)+$ Hom $\left[\pi_{1}(B), G / N\right]$ according as $B$ is orientable or not. ( $N$ is the arcwise component of the identity in $G$; a slight additional hypothesis is needed for the nonorientable case.) (2) Let $O^{n}$ be the group of all orthogonal transformations of $S^{n}$; let $p=$ genus of $B(p=2-\chi$ if $B$ is nonorientable). Then the bundles fall into $2^{2 p}$ or $2^{p}$ "types," according as $B$ is orientable or not. If $n>1$, each type includes exactly two abstract bundles. If $n=1$, the same holds except for one type, whose members are in 1-1 correspondence with the non-negative integers. (Received November 16,1950.)

184t. Mrs. B. R. Vanderslice and D. W. Hall: Chromatic polynomials. Preliminary report.

G. D. Birkhoff and D. C. Lewis, Jr. have made a conjecture (Trans. Amer. Math. Soc. vol. 60 (1946) pp. 355-451, in particular pp. 411-413) which, if true, will establish the four color conjecture in the affirmative. This paper is a first step towards the experimental verification of the Birkhoff-Lewis conjecture for the special case of the truncated icosahedron. This particular map is of interest since it is the simplest map with triple vertices containing no region of fewer than five sides and having the property that its pentagons are mutually isolated. In the work towards the computation of the chromatic polynomial for the truncated isocahedron, the polynomials have already been obtained for several hundred new maps, quite a few of which contain more than twenty regions. (Received November 14,1950.)

\section{A. D. Wallace: Rim compact spaces.}

The space $X$ is rim compact at $A \subset X$ if each neighborhood of $A$ contains a neighborhood of $A$ with a compact boundary. We term $X$ rim compact (RC) if it is RC at each of its points. Let now $X$ be any RCH space. It is shown, among other things, that: (i) $X$ is RC at any of its closed sets with a compact boundary; (ii) $X$ is completely regular; (iii) any quasicomponent of $X$ with a compact boundary is also a component. We remark that, under different names, RC spaces have been investigated by Freudenthal, Jones, Zippin, and others. (Received November 13, 1950.) 


\section{APPENDIX}

\section{EXCERPTS FROM REPORT OF TREASURER}

December 20, 1950

To the Board of Trustees of the

American Mathematical Society

Gentlemen:

I have the honor to submit herewith the report of the Treasurer for the fiscal year ended November 30,1950, with certain pertinent comments.

\section{Investment Portfolio and Income}

On November 30, 1950, the market value of securities held for Invested Funds exceeded book value by $\$ 36,000$ while the market value of securities held for Current Funds was less than book value by just over $\$ 100$. On the whole portfolio, the market value therefore exceeds book value by $\$ 35,900$. Reserves held in accounts "Reserve for Investment Losses" $(\$ 4,386)$ and "Profit on Sales of Securities" $(\$ 18,168)$ may still be considered adequate protection against contingent depreciation in market value.

The following is a summary of the changes in the security holdings made during the year.

Acquired

$\$ 16,100$ U.S. Treasury $2 \frac{1}{2} \mathrm{~s} 1957-52$

100 shares Consumers Power Co. common

50 shares Goodyear Tire and Rubber Co. cum. pref. $\$ 5.00$

100 shares Niagara Mohawk Power Co. common

60 shares U. S. Steel Corp. 7\% cum. pref.

10 shares U. S. Smelting, Refining and Mining Co. $7 \%$ cum. pref.

Sold

$\$ 7,000$ U. S. Treasury 2s $1954-52$

30 Aetna Insurance Co. common

100 rights Consumers Power Co. common

100 shares Crane Co. common

100 shares International Nickel Co. of Canada common

15 shares National Biscuit Co. 7\% cum. pref.

200 shares National Dairy common

100 shares Owens Illinois Glass Co. common 
53 shares Texas Company common

50 shares Union Oil Company of California $\$ 3.75$ cum. pref.

Redeemed

\section{$\$ 10,000$ U. S. Savings Bonds}

The investment portfolio, valued at market November 30, 1950, now includes Government bonds 33 per cent, other bonds 8 per cent, preferred stock 18 per cent, and common stock 41 per cent.

All savings accounts of the Society have been closed and the money used to meet current obligations. These accounts represented accumulated unspent income from earlier years.

Income received during the year from investment of Current Funds amounted to $\$ 1,837$. This represents a return of 2.3 per cent computed on average book value of investments. Income on Invested Funds amounted to $\$ 10,859$, representing a return of 5.6 per cent. Total investment income from all sources was $\$ 12,696$, representing a return of over 4.3 per cent.

Income from the Henderson Estate was $\$ 6,025$; in 1949 it was $\$ 5,200$. It may be noted that the Society's share in the income of this estate has now increased by $\$ 500$ annually, as one of the other beneficiaries died during the year.

During the year the Society received the principal of the Helen A. Merrill Bequest, securities valued at $\$ 650$. By action of the Trustees, this has been set up as a special fund. There are probably a number of members of the Society who are in a position to include the Society among the beneficiaries of their estates, either by bequest of a specific sum or by ultimately turning over to the Society funds which have been used to provide a life trust for a relative. The importance of such bequests, even in small amounts, cannot be overestimated. The officers of the Society will be glad to furnish information as to the legal form for such bequests.

\section{The General Financial Picture}

A brief summary of the scope of the Society's program may be of interest. The annual general receipts of the Society are just short of $\$ 65,000$. The expenses of maintaining the Society's offices, holding meetings, and generally conducting its affairs come to approximately $\$ 32,000$. The Bulletin, Proceedings, and Transactions are regarded as general publications of the Society, whereas all other publications are charged against special publication funds set up for the purpose. The annual cost of the Bulletin, Proceedings, and Transac- 
tions is about $\$ 48,000$. Clearly, therefore, the Society is annually depleting its reserves to a serious extent. Either additional sources of income must be found, or the scope of the Society's activity must be curtailed.

The recent increase in dues will probably make it possible to support the general Society activities, including publication of the Bulletin through general receipts. The Proceedings, though sent to each member, are-like the Transactions-more specialized in character than the Bulletin and it would seem logical that special appropriations, which might in whole or in part come from general funds, rather than unspecialized income, be used for their support.

Apparently, the assets of the Society have increased by more than $\$ 17,000$. The increase is fictitious. The International Congress account has increased by more than $\$ 27,000$ - money taken in at the Congress which is being held by the Society to meet the further expenses of the Congress, including the publication of the Proceedings. Furthermore, the Society has not yet been billed for all of the publications properly chargeable to the business of fiscal 1950. It is estimated that these bills will amount to $\$ 10,000$.

Except for the use of $\$ 10,840$ in its savings accounts and $\$ 10,000$ from redeemed U. S. Savings Bonds the Society was not compelled this year to liquidate any of the investments of current funds. In effect we have borrowed approximately $\$ 12,000$ from the International Congress Fund to obtain cash to meet the ordinary expenses of the Society.

The situation remains critical and the Treasurer cannot but urge the Trustees to give full and earnest consideration to the difficulties faced by the Society because of its lack of sufficient income to discharge the obligations placed upon it by the growth and vitality of American mathematics.

Respectfully submitted, Albert E. Meder, JR., Treasurer 
BALANCE SHEET

Assets

Current Funds:

November 30, November 30 ,

1950

1949

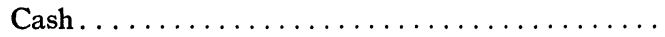

Due from Invested Funds.

$\$ 33,332.51 \$ 12,057.26$

Account Receivable from United States Government..........................

Investments. . . . . . . . . . . . . . . . .

\begin{tabular}{|c|c|}
\hline $\begin{array}{r}5,842.66 \\
67,328.66\end{array}$ & $\begin{array}{r}3,440.49 \\
76,292.42\end{array}$ \\
\hline$\$ 106,503.83$ & $\$ 91,821.75$ \\
\hline
\end{tabular}

INVESTED Funds:

Cash. . . . . . . . . . . . . . . . . . . .

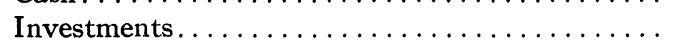

$\$ 629.83$

$194,242.32 \quad 192,153.46$

$\$ 194,872.15 \$ 192,153.46$

Total Assets.

$\$ 301,375.98 \quad \$ 283,975.21$

\section{Current Funds:}

\section{Liabilities}

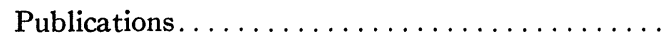

International Congress...................

Policy Committee

Prize Funds and Other Special Funds Accumulated

Income. . . . . . . . . . . . . . . . . . . . .

Sinking Fund $\ldots \ldots \ldots \ldots \ldots \ldots \ldots \ldots \ldots$

Profit on Sales of Securities.................

Miscellaneous........................

Surplus ...........................

\begin{tabular}{rr}
$\$ 26,348.27$ & $\$ 23,928.48$ \\
$45,531.48$ & $18,089.17$ \\
$(648.55)$ & $(160.70)$ \\
& \\
$3,524.71$ & $9,553.82$ \\
54.04 & $1,244.97$ \\
$2,049.85$ & $2,032.98$ \\
$1,644.02$ & 986.90 \\
$28,000.01$ & $36,146.13$ \\
\hline$\$ 106,503.83$ & $\$ 91,821.75$ \\
\hline
\end{tabular}

\section{INVESTED FUNDS:}

Endowment Fund Principal. ..............

Prize Funds and Other Special Funds..........

Life Membership and Subscription Reserve......

Mathematical Reviews...................

Reserve for Investment Losses. . . . . . . . . . . . . . . .

Profit on Sales of Securities.................

Due to Current Funds.

$$
\begin{array}{rrr}
\$ 71,000.00 & \$ 71,000.00 \\
33,683.22 & 33,033.22 \\
2,635.08 & 2,851.70 \\
65,000.00 & 65,000.00 \\
4,385.89 & 4,385.89 \\
18,167.96 & 15,851.07 \\
& & 31.58 \\
\hline & & \$ 192,153.46 \\
\hline 194,872.15 & & \\
\cline { 1 - 1 } \$ 301,375.98 & & \$ 283,975.21
\end{array}
$$

Total Liabilities 


\section{SUMMARY STATEMENT OF INCOME AND EXPENDITURES}

$$
\text { 1949-1950 }
$$

General Receipts

1950

Dues-Ordinary Memberships.

Dues-Contributing Memberships.......

$\$ 33,010 \$ 30,017$

Dues-Institutional Memberships.......

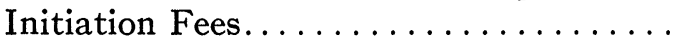

Investment Income. . . . . . . . . . . 13,171

Miscellaneous.

\begin{tabular}{|c|c|}
\hline 1,517 & 122 \\
\hline$\$ 65,152$ & $\$ 53,910$ \\
\hline $93,507^{1}$ & $73,775^{2}$ \\
\hline 5,545 & 1,884 \\
\hline$\$ 164,204$ & $\$ 129,569$ \\
\hline
\end{tabular}

Publications Receipts

TOtal Receipts.

General Expense.

Total Expenses..............

1 This includes $\$ 21,500$ received under a contract with the Office of Air Research for support of Mathematical Reviews.

${ }^{2}$ This includes $\$ 7,140$ received under a contract with the Office of Naval Research for support of Mathematical Reviews. 